\title{
Tight inefficiency bounds for perception-parameterized affine congestion games
}

\author{
Pieter Kleer ${ }^{\mathrm{a}, 1}$, Guido Schäfer ${ }^{\mathrm{a}, \mathrm{b}, *}$ \\ a Centrum Wiskunde E Informatica (CWI), Amsterdam, the Netherlands \\ b Vrije Universiteit Amsterdam, Amsterdam, the Netherlands
}

\section{A R T I C L E I N F O}

\section{Article history:}

Received 10 September 2017

Received in revised form 19 March 2018

Accepted 13 April 2018

Available online $\mathrm{xxxx}$

\section{Keywords:}

Congestion games

Inefficiency of equilibria

Price of anarchy

Price of stability

Altruistic games

Universal taxes

Risk-averse players

\begin{abstract}
A B S T R A C T
We introduce a new model of congestion games that captures several extensions of the classical congestion game introduced by Rosenthal in 1973. The idea here is to parameterize both the perceived cost of each player and the social cost function of the system designer. Intuitively, each player perceives the load induced by the other players by an extent of $\rho \geq 0$, while the system designer estimates that each player perceives the load of all others by an extent of $\sigma \geq 0$. For specific choices of $\rho$ and $\sigma$, we obtain extensions such as altruistic player behavior, risk sensitive players and the imposition of taxes on the resources. We derive tight bounds on the price of anarchy and the price of stability for a large range of parameters. Our bounds provide a complete picture of the inefficiency of equilibria for these games. As a result, we obtain tight bounds on the price of anarchy and the price of stability for the above mentioned extensions. Our results also reveal how one should "design" the cost functions of the players in order to reduce the price of anarchy. Somewhat counterintuitively, if each player cares about all other players to the extent of $\rho=0.625$ (instead of 1 in the standard setting) the price of anarchy reduces from 2.5 to 2.155 and this is best possible.
\end{abstract}

(c) 2018 Elsevier B.V. All rights reserved.

\section{Introduction}

Congestion games constitute an important class of non-cooperative games which was introduced by Rosenthal in 1973 [19]. In a congestion game, we are given a set of resources from which a set of players can choose. Each resource is associated with a cost function which specifies the cost of this resource depending on the total number of players using it. Every player chooses a subset of resources (from a set of resource subsets available to her) and experiences a cost equal to the sum of the costs of the chosen resources. Congestion games are both theoretically appealing and practically relevant. For example, they have applications in network routing, resource allocation and scheduling problems.

Rosenthal [19] proved that every congestion game has a pure Nash equilibrium, i.e., a strategy profile such that no player can decrease her cost by unilaterally deviating to another feasible set of resources. This result was established through the use of an exact potential function (known as Rosenthal potential) satisfying that the cost difference induced by a unilateral player deviation is equal to the potential difference of the respective strategy profiles. In fact, Monderer and Shapley [17] showed that the class of games admitting an exact potential function is isomorphic to the class of congestion games.

\footnotetext{
* Corresponding author.

E-mail addresses: kleer@cwi.nl (P. Kleer), schaefer@cwi.nl (G. Schäfer).

1 Pieter Kleer is supported by the NWO Gravitation Project NETWORKS, Grant Number 024.002.003.
} 
Table 1

An overview of our (tight) price of anarchy and price of stability results for certain values of $\rho$ and $\sigma$. Here $h(1) \approx 0.625$ (see Theorem 2 for a formal definition). The respective references where these bounds were established first are given in the column "Ref."; an asterisk indicates that this result is new.

\begin{tabular}{|c|c|c|c|c|c|}
\hline Model & Parameters & PoA & Ref. & PoS & Ref. \\
\hline Classical & $\rho=\sigma=1$ & $\frac{5}{2}$ & [8] & 1.577 & {$[4]$} \\
\hline Altruism (1) & $\sigma=1,1 \leq \rho \leq 2$ & $\frac{4 \rho+1}{1+\rho}$ & {$[5,6]$} & $\frac{\sqrt{3}+1}{\sqrt{3}+\rho-1}$ & {$\left[{ }^{*}\right]$} \\
\hline Altruism (2) & $\sigma=1,2 \leq \rho \leq \infty$ & $\rho+1$ & {$[6]$} & - & - \\
\hline Risk neutral-players & $\sigma=\rho=\frac{1}{2}$ & $\frac{5}{3}$ & [18] & 1.447 & {$\left[{ }^{*}\right]$} \\
\hline Wald's minimax & $\sigma=\frac{1}{2}, \rho=1$ & 2 & {$[3,18]$} & 1 & {$\left[{ }^{*}\right]$} \\
\hline Constant universal taxes & $\sigma=1, \rho=h(1)$ & 2.155 & {$[4]$} & 2.013 & {$[*]$} \\
\hline Uniform affine CG & - & $\infty$ & {$\left[{ }^{*}\right]$} & 2 & {$\left[{ }^{*}\right]$} \\
\hline
\end{tabular}

One of the main research directions in algorithmic game theory focuses on quantifying the inefficiency caused by selfish behavior. The idea is to assess the quality of a Nash equilibrium relative to an optimal outcome. Here the quality of an outcome is measured in terms of a given social cost objective (e.g., the sum of the costs of all players). Koutsoupias and Papadimitriou [14] introduced the price of anarchy as the worst-case ratio between the social cost of a Nash equilibrium and the social cost of an optimum. Anshelevich et al. [1] defined the price of stability as the ratio between the best social cost of a Nash equilibrium and the social cost of an optimum.

In recent years, several extensions of Rosenthal's congestion games were proposed to incorporate aspects which are not captured by the standard model. For example, these extensions include risk sensitivity of players in uncertain settings [18], altruistic player behavior [5,6] and congestion games with taxes [4]. We elaborate in more detail on these extensions in Section 2. These games were studied intensively with the goal to obtain a precise understanding of the price of anarchy.

In this paper, we introduce a new model of congestion games, which we term perception-parameterized congestion games, that captures all these extensions (and more) in a unifying way. The key idea here is to parameterize both the perceived cost of each player and the social cost function. Intuitively, each player perceives the load induced by the other players by an extent of $\rho \geq 0$, while the system designer estimates that each player perceives the load of all others by an extent of $\sigma \geq 0$. The above mentioned extensions reduce to special cases of our model by choosing the parameters $\rho$ and $\sigma$ accordingly.

We illustrate our model by means of a simple example; formal definitions of our perception-parameterized congestion games are given in Section 2. Suppose we are given a set of $m$ resources and that every player has to choose precisely one of these resources. The cost of a resource $e$ is given by a cost function $c_{e}$ that maps the load on $e$ to a real value. In the classical setting, the load of a resource $e$ is defined as the total number of players $x_{e}$ using $e$. That is, the cost that player $i$ experiences when choosing resource $e$ is $c_{e}\left(x_{e}\right)$. In contrast, in our setting players have different perceptions of the load induced by the other players. More precisely, the perceived load of player $i$ choosing resource $e$ is $1+\rho\left(x_{e}-1\right)$, where $\rho \geq 0$ is some parameter. ${ }^{2}$ Consequently, the perceived cost of player $i$ for choosing $e$ is $c_{e}\left(1+\rho\left(x_{e}-1\right)\right)$. Note that as $\rho$ increases players care more about the presence of other players. In addition, we introduce a similar parameter $\sigma \geq 0$ for the social cost objective, i.e., the social cost is defined as $\sum_{e} c_{e}\left(1+\sigma\left(x_{e}-1\right)\right) x_{e}$. Intuitively, this can be seen as the system designer's estimate of how each player perceives the load of the other players.

Despite the fact that we deal with a more general class of congestion games, we manage to derive tight bounds on the price of anarchy and the price of stability for a large range of parameters $\rho$ and $\sigma$. Our bounds provide a complete picture of the inefficiency of equilibria for these perception-parameterized congestion games. As a consequence, we obtain tight bounds on the price of anarchy and the price of stability for the above mentioned extensions. While the price of anarchy bounds are (mostly) known from previous results, the price of stability results are new. As in [4-6,18], we focus on congestion games with affine cost functions.

Our results We prove the following bounds on the price of anarchy (PoA) and the price of stability (PoS) of pure Nash equilibria in affine congestion games for a large range of parameters $(\rho, \sigma)$ (specified below):

$$
\operatorname{PoA} \leq \max \left\{\rho+1, \frac{2 \rho(1+\sigma)+1}{\rho+1}\right\} \quad \text { and } \quad \operatorname{PoS} \leq \frac{\sqrt{\sigma(\sigma+2)}+\sigma}{\sqrt{\sigma(\sigma+2)}+\rho-\sigma} .
$$

We prove that these bounds are tight for general affine congestion games. Further, for the special case of symmetric network congestion games we show that the bound of $(2 \rho(1+\sigma)+1) /(\rho+1)$ on the price of anarchy is asymptotically tight. In contrast, for this case we derive a better (tight) bound on the price of stability for $\sigma=1$ and $\rho \geq 0$. An overview of the price of anarchy and the price of stability results that we obtain from (1) for several applications known in the literature is given in Table 1; see Fig. 1 for an illustration of our PoA bound. The connection between these applications and our model is discussed at the end of Section 2.

In light of the above bounds, we obtain an (almost) complete picture of the inefficiency of equilibria (parameterized by $\rho$ and $\sigma$ ). For example, see Fig. 2 for an illustration of the price of anarchy for $\sigma=1$. Note that the price of anarchy decreases

\footnotetext{
2 In general, the perception parameter $\rho$ might be player-specific. Here we focus on the homogeneous player case and leave the heterogenous case for future work (see concluding remarks). 


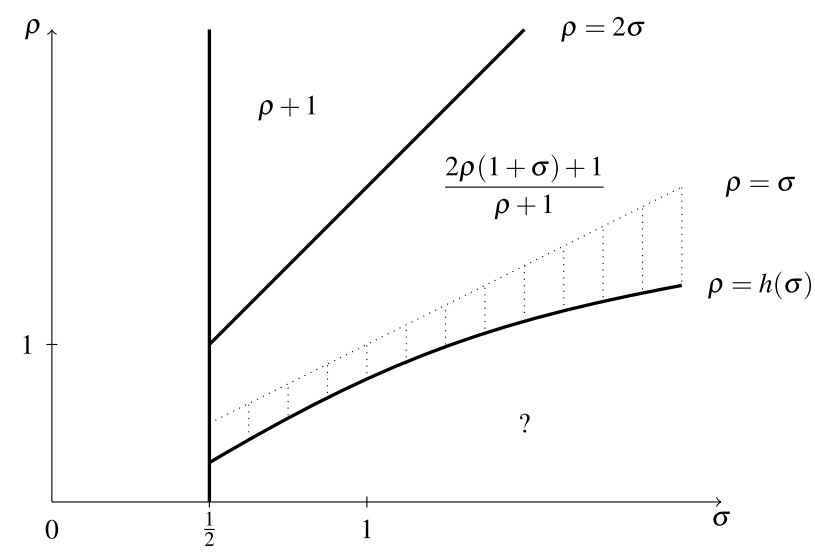

Fig. 1. The bound $\rho+1$ holds for $\rho \geq 2 \sigma \geq 1$. The bound $(2 \rho(1+\sigma)+1) /(1+\rho)$ holds for $\sigma \leq \rho \leq 2 \sigma$. Basically, this bound also holds for $h(\sigma) \leq \rho \leq \sigma$, but our proof of Theorem 2 only works for a discretized range of $\sigma$ (hence the vertical dotted lines in this area). The function $h$ is given in Theorem 2 .

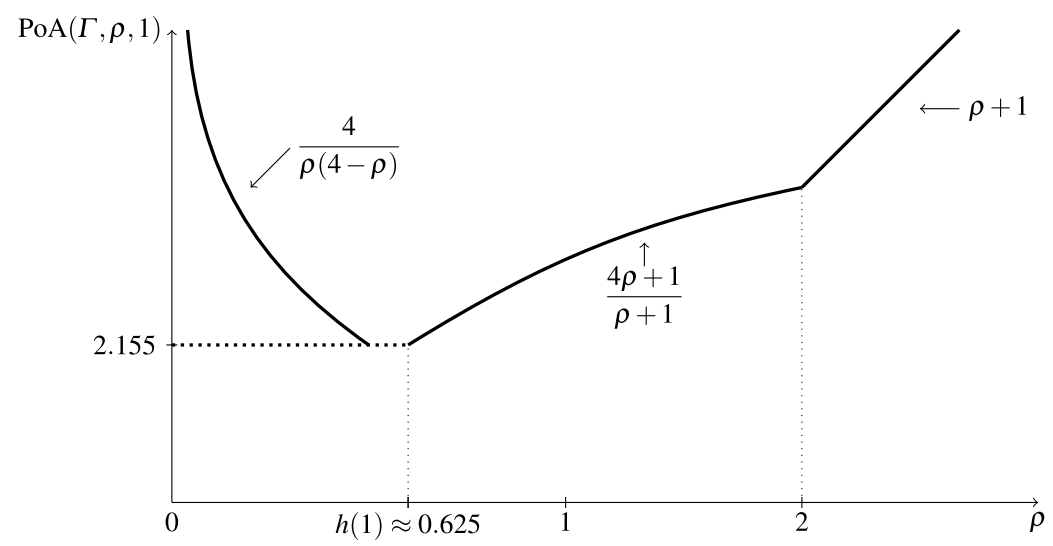

Fig. 2. Lower bounds on the price of anarchy for $\sigma=1$. The bounds $(4 \rho+1) /(\rho+1)$ and $\rho+1$ are also tight upper bounds. The dotted horizontal line indicates the lower bound following from [5, Theorem 3.7]. The bound $4 /(\rho(4-\rho))$ is a lower bound for symmetric singleton congestion games given in the proof of Theorem 7. A tight bound for $0<\rho \leq h(1)$ remains an open problem.

from $\frac{5}{2}$ for $\rho=1$ to 2.155 for $\rho=h(1) \approx 0.625$. The price of anarchy for $\rho=h(1)$ was first established by Caragiannis et al. [4]. Note that our bounds imply that the price of anarchy is in fact minimized at $\rho=h(1)$ (see also Fig. 2). In particular, this shows that the bound of $(4 \rho+1) /(\rho+1)$ proven in [6] for $1 \leq \rho \leq 2$ continues to hold for $h(1) \leq \rho \leq 1$. This nicely bridges the results in [4] and [6].

\section{Our model and applications}

In this section, we first formally introduce our model of congestion games with parameterized perceptions (Section 2.1). We then show that our model subsumes several other models that were studied in the literature as special cases (Section 2.2).

\subsection{Perception-parameterized congestion games}

A congestion game $\Gamma$ is given by a tuple $\left(N, E,\left(\mathcal{S}_{i}\right)_{i \in N},\left(c_{e}\right)_{e \in E}\right)$, where $N=[n]^{3}$ is a (finite) set of players, $E$ is a (finite) set of resources (or facilities), $\mathcal{S}_{i} \subseteq 2^{E}$ is a set of strategies of player $i$, and $c_{e}: \mathbb{R}_{\geq 0} \rightarrow \mathbb{R}_{\geq 0}$ is a cost function of facility $e$. Given a strategy profile $s=\left(s_{1}, \ldots, s_{n}\right) \in \times_{i} \mathcal{S}_{i}$, we define $x_{e}$ as the number of players using resource $e$, i.e., $x_{e}=x_{e}(s)=$ $\left|\left\{i \in N: e \in s_{i}\right\}\right|$. We also call $x=\left(x_{e}\right)_{e \in E}$ the load profile of $s$. If $\mathcal{S}_{i}=\mathcal{S}_{j}$ for all $i, j \in N$, the game is called symmetric. For a given graph $G=(V, E)$, we call $\Gamma$ a (directed) network congestion game if for every player $i$ there exist $s_{i}, t_{i} \in V$ such that $\mathcal{S}_{i}$ is the set of all (directed) $\left(s_{i}, t_{i}\right)$-paths in $G$. An affine congestion game has cost functions of the form $c_{e}(x)=a_{e} x+b_{e}$ with $a_{e}, b_{e} \geq 0$. If $b_{e}=0$ for all $e \in E$, the game is called linear.

${ }^{3}$ Given a positive integer $n$, we use $[n]$ to refer to the set $\{1, \ldots, n\}$. 
We introduce our unifying model of perception-parameterized congestion games with affine cost functions. For a fixed parameter $\rho \geq 0$, we define the cost of player $i \in N$ by

$$
C_{i}^{\rho}(s)=\sum_{e \in S_{i}} c_{e}\left(1+\rho\left(x_{e}-1\right)\right)=\sum_{e \in s_{i}} a_{e}\left[1+\rho\left(x_{e}-1\right)\right]+b_{e}
$$

for a given strategy profile $s=\left(s_{1}, \ldots, s_{n}\right)$. For a fixed parameter $\sigma \geq 0$, the social cost of a strategy profile $s$ is given by

$$
C^{\sigma}(s)=\sum_{i \in N} C_{i}^{\sigma}(s)=\sum_{e \in E} x_{e}\left(a_{e}\left[1+\sigma\left(x_{e}-1\right)\right]+b_{e}\right) .
$$

We refer to the case $\rho=\sigma=1$ as the classical congestion game setting (with cost functions $c_{e}(x)=a_{e} x+b_{e}$ for all $e \in E$ and social cost function $C(s)=\sum_{i} C_{i}(s)$.)

A strategy profile $s$ is a pure Nash equilibrium if for all players $i \in N$ it holds that $C_{i}^{\rho}(s) \leq C_{i}^{\rho}\left(s_{i}^{\prime}, s_{-i}\right)$ for all $s_{i}^{\prime} \in \mathcal{S}_{i}$, where $\left(s_{i}^{\prime}, s_{-i}\right)$ denotes the strategy profile in which player $i$ plays $s_{i}^{\prime}$ and all the other players their strategy in $s$.

The price of anarchy $(P O A)$ and the price of stability (POS) of a game $\Gamma$ are defined as

$$
\operatorname{PoA}(\Gamma, \rho, \sigma)=\frac{\max _{s \in \mathrm{NE}} C^{\sigma}(s)}{\min _{s^{*} \in x_{i} \mathcal{S}_{i}} C^{\sigma}\left(s^{*}\right)} \text { and } \operatorname{PoS}(\Gamma, \rho, \sigma)=\frac{\min _{s \in \mathrm{NE}} C^{\sigma}(s)}{\min _{s^{*} \in x_{i}} \mathcal{S}_{i} C^{\sigma}\left(s^{*}\right)},
$$

where $\mathrm{NE}=\mathrm{NE}(\rho)$ denotes the set of pure Nash equilibria with respect to the player costs as defined in (2). For a collection of games $\mathcal{H}$,

$$
\operatorname{PoA}(\mathcal{H}, \rho, \sigma)=\sup _{\Gamma \in \mathcal{H}} \operatorname{PoA}(\Gamma, \rho, \sigma) \text { and } \operatorname{PoS}(\mathcal{H}, \rho, \sigma)=\sup _{\Gamma \in \mathcal{H}} \operatorname{PoS}(\Gamma, \rho, \sigma) .
$$

Unless stated otherwise, our results refer to the class of perception-parameterized congestion games with affine cost functions; we therefore drop the parameter $\mathcal{H}$ below.

Rosenthal [19] shows that every congestion game admits an exact potential function, i.e., a function $\Phi: \times_{i} \mathcal{S}_{i} \rightarrow \mathbb{R}$ such that for every strategy profile $s$, for every $i \in N$ and every $s_{i}^{\prime} \in \mathcal{S}_{i}$ :

$$
\Phi(s)-\Phi\left(s_{-i}, s_{i}^{\prime}\right)=C_{i}(s)-C_{i}\left(s_{-i}, s_{i}^{\prime}\right) .
$$

More specifically, given a congestion game $\Gamma$ with arbitrary cost functions $\left(\bar{c}_{e}\right)_{e \in E}$, the Rosenthal potential (see [19])

$$
\Phi(s)=\sum_{e \in E} \sum_{k=1}^{x_{e}} \bar{c}_{e}(k)
$$

is an exact potential function.

In particular, for every fixed parameter $\rho \geq 0$ the Rosenthal potential (4) with cost functions as defined in (2), i.e.,

$$
\bar{c}_{e}(x)=c_{e}(1+\rho(x-1))=a_{e}[1+\rho(x-1)]+b_{e},
$$

is an exact potential function for the corresponding perception-parameterized congestion game. As a consequence, pure Nash equilibria always exist for these games.

\subsection{Applications}

We review various models that fall within our model of perception parameterized congestion games introduced above (for certain values of $\rho$ and $\sigma$ ).

Altruism [5,6]. We can rewrite the cost of player $i$ as

$$
C_{i}^{\rho}(s)=\sum_{e \in s_{i}}\left(a_{e} x_{e}+b_{e}\right)+(\rho-1) a_{e}\left(x_{e}-1\right) .
$$

The term $(\rho-1) a_{e}\left(x_{e}-1\right)$ can be interpreted as a "dynamic" (meaning load-dependent) tax that all players using resource $e$ have to pay. For $1 \leq \rho \leq \infty$ and $\sigma=1$, this model is equivalent to the altruistic player setting proposed by Caragiannis et al. [5]. Chen et al. [6] also study this model of altruism for $1 \leq \rho \leq 2$ and $\sigma=1{ }^{4}$

4 The equivalence between the altruism model in [5] and our model is immediate; the equivalence between the altruism model in [6] and the model in [5] (and thus also our model) is proven in [6]. 
Universal taxes [4]. We can rewrite the cost of player $i$ as

$$
C_{i}^{\rho}(s)=\sum_{e \in s_{i}} \rho a_{e} x_{e}+(1-\rho) a_{e}+b_{e} .
$$

Dividing by $\rho$ gives that $s$ is a Nash equilibrium with respect to $C_{i}^{\rho}$ if and only if $s$ is a Nash equilibrium with respect to

$$
T_{i}^{\rho}(s)=\frac{C_{i}^{\rho}}{\rho}=\sum_{e \in s_{i}}\left(a_{e} x_{e}+\frac{b_{e}}{\rho}\right)+\sum_{e \in s_{i}} \frac{1-\rho}{\rho} a_{e} .
$$

That is, $s$ is a Nash equilibrium in a classical congestion game in which players take into account constant resource taxes of the form $\tau(\rho) \cdot a_{e}$, where $\tau(\rho)=(1-\rho) / \rho$. Caragiannis, Kaklamanis and Kanellopoulos [4] study this type of taxes, which they call universal tax functions, for $\rho$ satisfying $\tau(\rho)=\frac{3}{2} \sqrt{3}-2$. They consider these taxes to be refundable, i.e., they are not taken into account in the social cost, which is equivalent to the case $\sigma=1$. Note that the function $\tau:(0,1] \rightarrow[0, \infty)$ with $\tau(\rho)=(1-\rho) / \rho$ is bijective. That is, there is a one-to-one correspondence between universal taxes with $\tau(\rho) \in[0, \infty)$ and spiteful behavior with $\rho \in(0,1]$; this relation is also mentioned by Caragiannis et al. [5].

Risk sensitivity under uncertainty [18]. Nikolova, Piliouras and Shamma [18] consider congestion games in which there is a (non-deterministic) order of the players on every resource. A player is only affected by players in front of her. That is, the load on resource $e$ for player $i$ in a strict ordering $r$, where $r_{e}(i)$ denotes the position of player $i$, is given by $x_{e}(i)=\left|\left\{j \in N: r_{e}(j) \leq r_{e}(i)\right\}\right|$. The cost of player $i$ is then $C_{i}(s)=\sum_{e \in s_{i}} c_{e}\left(x_{e}(i)\right)$. Note that $x_{e}(i)$ is a random variable if the ordering is non-deterministic. The social cost of the model is defined by the sum of all player costs

$$
C^{\frac{1}{2}}(s)=\sum_{e \in E} \frac{1}{2} a_{e} x_{e}\left(x_{e}+1\right)+b_{e}
$$

which is independent of the ordering $r .{ }^{5}$ Note that the social cost corresponds to the case $\sigma=\frac{1}{2}$ in our framework. Nikolova et al. [18] study various risk attitudes towards the ordering $r$ that is assumed to have a uniform distribution over all possible orderings. In particular, they consider players who are risk-neutral and players who apply Wald's minimax principle. In the risk-neutral setting the cost of a player is defined as the expected cost under the ordering $r$, which corresponds to the case $\rho=\frac{1}{2}$ in (2). Intuitively, this can be interpreted as that players expect to be scheduled in the middle on average. In contrast, when players apply Wald's minimax principle they adopt a worst-case perspective, i.e., each player assumes that she is scheduled last on all the resources; this corresponds to the case $\rho=1$.

Approximate Nash equilibria [9]. Suppose that $s$ is a Nash equilibrium under the cost functions defined in (2). Then, in particular, we have

$$
C_{i}^{1}(s) \leq C_{i}^{\rho}(s) \leq C_{i}^{\rho}\left(s_{i}^{\prime}, s_{-i}\right) \leq \rho C_{i}^{1}\left(s_{i}^{\prime}, s_{-i}\right)
$$

for any player $i$ and $s_{i}^{\prime} \in \mathcal{S}_{i}$ and $\rho \geq 1$. That is, we have $C_{i}^{1}(s) \leq \rho C_{i}^{1}\left(s_{i}^{\prime}, s_{-i}\right)$ which means that the profile $s$ is a $\rho$-approximate equilibrium, as studied by Christodoulou, Koutsoupias and Spirakis [9]. In particular, this implies that any upper bound on the price of anarchy, or price of stability, in our framework yields an upper bound on the price of stability for $\rho$-approximate equilibria for the same class of games.

Uniform affine congestion games. Let $\mathcal{A}^{\prime}$ denote the class of all congestion games $\Gamma$ for which all resources have uniform costs $c(x)=a x+b$, where $a=a(\Gamma)$ and $b=b(\Gamma)$ satisfy $a \geq 0$ and $a+b>0$. Note that we allow $b$ to be negative here. The class of affine congestion games with non-negative coefficients as defined above is contained in $\mathcal{A}^{\prime}$ since every such game can always be transformed ${ }^{6}$ into a game $\Gamma^{\prime}$ with $a_{e}=1$ and $b_{e}=0$ for all resources $e \in E^{\prime}$, where $E^{\prime}$ is the resource set of $\Gamma^{\prime}$. Without loss of generality, we can assume that $a+b=1$ (since the cost functions can be scaled by $1 /(a+b)$ ). The cost functions of $\Gamma \in \mathcal{A}^{\prime}$ can then equivalently be written as $c(x)=\rho x+(1-\rho)$ for $\rho \geq 0$. This is precisely the definition of $C_{i}^{\rho}(s)$ (with $a_{e}=1$ and $b_{e}=0$ ). In particular, if we take $\sigma=\rho$ (and thus $C^{\rho}(s)=\sum_{i \in N} C_{i}^{\rho}(s)$ ), we have

$$
\operatorname{PoA}\left(\mathcal{A}^{\prime}\right)=\sup _{\rho \geq 0} \operatorname{PoA}(\mathcal{A}, \rho, \rho) \quad \text { and } \quad \operatorname{PoS}\left(\mathcal{A}^{\prime}\right)=\sup _{\rho \geq 0} \operatorname{PoS}(\mathcal{A}, \rho, \rho),
$$

where $\mathcal{A}$ denotes the class of affine congestion games with non-negative coefficients.

The structure of the paper is as follows: In Section 3 we present our bounds on the price of anarchy for perceptionparameterized congestion games. In Section 4 we give the bounds on the price of stability. Finally, in Section 5 we comment on the implications of our results on the models mentioned above and derive some additional insights.

\footnotetext{
5 In every ordering there is always one player first, one player second, and so on.

6 This transformation can be done in such a way that both the PoA and the PoS of the game do not change; see, e.g., [6, Lemma 4.3] for a proof.
} 


\section{Price of anarchy}

In this section, we present our bounds on the price of anarchy; see Fig. 1 for an illustration. We first start with the simpler proof of the $\rho+1$ bound (Section 3.1) and then turn to the more involved proof of the $(2 \rho(1+\sigma)+1) /(\rho+1)$ bound (Section 3.2). Both bounds are shown to be tight for affine congestion games. For the latter bound, we prove that it is asymptotically tight even for the special case of symmetric network congestion games (Section 3.3).

We need the following technical lemma.

Lemma 1. Let $\rho, \sigma \geq 0$ be fixed. If there exist $\alpha:=\alpha(\rho, \sigma) \geq 0$ and $\beta:=\beta(\rho, \sigma)>0$ such that for all non-negative integers $x$ and $y$ the inequality

$$
(1+\rho x) y-\rho(x-1) x-x \leq-\beta(1+\sigma(x-1)) x+\alpha(1+\sigma(y-1)) y
$$

holds, then $\operatorname{PoA}(\rho, \sigma) \leq \alpha / \beta$.

Proof. Without loss of generality, we may assume that $a_{e}=1$ and $b_{e}=0$ for all resources $e \in E$ (see, e.g., [6, Lemma 4.3]). Let $s$ be a Nash equilibrium with respect to the cost functions $C_{i}^{\rho}(s)$ and let $s^{*}$ be a minimizer of $C^{\sigma}(\cdot)$. Further, let $x$ and $x^{*}$ be the load profiles for $s$ and $s^{*}$, respectively.

We have

$$
\begin{aligned}
\sum_{i} C_{i}^{\rho}(s) & =\sum_{e} \rho\left(x_{e}-1\right) x_{e}+\sum_{e} x_{e} \\
& =\sum_{e} \rho[1-\sigma+\sigma]\left(x_{e}-1\right) x_{e}+\rho x_{e}-\rho x_{e}+\sum_{e} x_{e} \\
& =\rho \sum_{e}\left[1+\sigma\left(x_{e}-1\right)\right] x_{e}+\rho \sum_{e}(1-\sigma)\left(x_{e}-1\right) x_{e}-x_{e}+\sum_{e} x_{e} \\
& =\rho C^{\sigma}(s)+\rho \sum_{e}(1-\sigma)\left(x_{e}-1\right) x_{e}+(1-\rho) \sum_{e} x_{e} .
\end{aligned}
$$

By rearranging terms, we obtain

$$
\begin{aligned}
\rho C^{\sigma}(s) & =\sum_{i} C_{i}^{\rho}(s)+\rho(\sigma-1) \sum_{e}\left(x_{e}-1\right) x_{e}+(\rho-1) \sum_{e} x_{e} \\
& \leq \sum_{i} C_{i}^{\rho}\left(s_{i}^{*}, s_{-i}\right)+\rho(\sigma-1) \sum_{e}\left(x_{e}-1\right) x_{e}+(\rho-1) \sum_{e} x_{e} \\
& \leq \sum_{e}\left[1+\rho\left(x_{e}-1+1\right)\right] x_{e}^{*}+\rho(\sigma-1) \sum_{e}\left(x_{e}-1\right) x_{e}+(\rho-1) \sum_{e} x_{e} \\
& =\sum_{e}\left[1+\rho x_{e}\right] x_{e}^{*}+\rho(\sigma-1)\left(x_{e}-1\right) x_{e}+(\rho-1) x_{e} \\
& =\sum_{e}\left[1+\rho x_{e}\right] x_{e}^{*}+\rho\left[1+\sigma\left(x_{e}-1\right)\right] x_{e}-\rho\left(x_{e}-1\right) x_{e}-x_{e} \\
& =\sum_{e}\left[1+\rho x_{e}\right] x_{e}^{*}-\rho\left(x_{e}-1\right) x_{e}-x_{e}+\rho C^{\sigma}(s) \\
& \leq-\beta C^{\sigma}(s)+\alpha C^{\sigma}\left(s^{*}\right)+\rho C^{\sigma}(s) .
\end{aligned}
$$

Here the first inequality holds because $s$ is a Nash equilibrium, the second inequality follows from the definition (2) and the last inequality holds because of (5). We conclude that $\beta C^{\sigma}(s) \leq \alpha C^{\sigma}\left(s^{*}\right)$, which proves the claim.

We remark that our upper bounds on the price of anarchy of perception-parameterized congestion games can alternatively be proven by adapting the smoothness framework of Roughgarden [20] appropriately; similarly as in [6].

\subsection{First PoA bound}

We establish the following tight bound on the price of anarchy:

Theorem 1. We have $\operatorname{PoA}(\rho, \sigma) \leq \rho+1$ for $1 \leq 2 \sigma \leq \rho$ and this bound is tight. 
Note that the bound itself does not depend on $\sigma$, only the range of $\rho$ and $\sigma$ for which it holds. For the altruism model of Caragiannis et al. [5] (corresponding to $\sigma=1$ and $\rho \geq 2$ ) this bound is known to be tight for non-symmetric singleton congestion games (i.e., all strategies consist of a single resource). Here we only prove tightness for general congestion games, but our construction is significantly simpler.

Proof of Theorem 1. By Lemma 1 it is sufficient to show that inequality (5) holds with $\beta=1$ and $\alpha=1+\rho$. Thus, we have to show that

$$
(1+\rho x) y-\rho(x-1) x-x \leq-(1+\sigma(x-1)) x+(1+\rho)(1+\sigma(y-1)) y .
$$

By rearranging terms, we obtain

$$
[y+\sigma y(y-1)-x y+x(x-1)] \rho+\sigma[y(y-1)-x(x-1)] \geq 0 .
$$

We first show that $[y+\sigma y(y-1)-x y+x(x-1)] \geq 0$ for all $\sigma \geq \frac{1}{2}$. It suffices to show this claim for $\sigma=\frac{1}{2}$, since $y(y-$ 1) $\geq 0$ for all $y \in \mathbb{N}$. We have

$$
y+\frac{1}{2} y(y-1)-x y+x(x-1)=\frac{1}{2}\left[\left(x-y-\frac{1}{2}\right)^{2}-\frac{1}{4}+x(x-1)\right]
$$

and this last expression is clearly non-negative for all $x, y \in \mathbb{N}$ (since the quadratic term is always at least $\frac{1}{4}$ ).

It now suffices to show (6) for $\rho=2 \sigma$, since we have shown that the expression is a non-decreasing affine function of $\rho$, for every fixed $\sigma \geq \frac{1}{2}$. Substituting $\rho=2 \sigma$ and dividing (6) by $\sigma$, we get the equivalent statement

$$
2[y+\sigma y(y-1)-x y+x(x-1)]+[y(y-1)-x(x-1)] \geq 0
$$

which we will show to be non-negative for all non-negative integers $x$ and $y$ and $\sigma \geq \frac{1}{2}$. Again, it suffices to show the statement for $\sigma=\frac{1}{2}$. The statement in (7) is then equivalent to

$$
\left(x-y-\frac{1}{2}\right)^{2}-\frac{1}{4}+y(y-1)
$$

which is clearly non-negative for all $x, y \in \mathbb{N}$.

To see that the bound is tight, consider the following game on four resources with two players: Player $A$ has strategies $\{\{1\},\{2,4\}\}$ and player $B$ has strategies $\left\{\{2\},\{1,3\}\right.$. Resources $e=1,2$ have cost function $c_{e}(x)=x$ and resources $e=3,4$ have cost function $c_{e}(x)=\rho x$. The optimum $s^{*}=(\{1\},\{2\})$ has cost 2 , whereas the Nash equilibrium $s=(\{2,4\},\{1,3\}$ has cost $2(1+\rho)$.

\subsection{Second PoA bound}

We next prove our $(2 \rho(1+\sigma)+1) /(\rho+1)$ bound on the price of anarchy. We first establish the upper bound for different ranges of parameters $\rho$ and $\sigma$.

\section{Theorem 2. We have}

$$
\operatorname{Po} A(\rho, \sigma) \leq \frac{2 \rho(1+\sigma)+1}{\rho+1}
$$

for

1. $\frac{1}{2} \leq \sigma \leq \rho \leq 2 \sigma$, or

2. $\sigma=1$ and $h(\sigma) \leq \rho \leq 2 \sigma$, where $h(\sigma)=g(1+\sigma+\sqrt{\sigma(\sigma+2)}, \sigma)$ is the maximum of the function

$$
g(a, \sigma)=\frac{\sigma\left(a^{2}-1\right)}{(1+\sigma) a^{2}-(2 \sigma+1) a+2 \sigma(\sigma+1)} .
$$

Further, there exists a function $\Delta=\Delta(\sigma)$ (specified in the proof below) satisfying that for every fixed $\sigma_{0} \geq \frac{1}{2}:$ if $\Delta\left(\sigma_{0}\right) \geq 0$, then the bound in (8) also holds for all $h\left(\sigma_{0}\right) \leq \rho \leq 2 \sigma_{0}$.

We need the following technical lemma in the proof of Theorem 2:

Lemma 2. Let $\sigma \geq \sigma^{*}:=\frac{1}{2}$ be fixed. Then for every $(x, y) \in \mathbb{N}^{2} \backslash\{(1,0)\}$, we have

$$
f_{1}(x, y, \sigma):=2 y(y-1) \sigma^{2}+\left[x^{2}+2 y^{2}-2 x y-x\right] \sigma+\left[x^{2}-x y+2(y-x)\right] \geq 0
$$


Proof. Note that $2 y(y-1) \geq 0$ for all $y \in \mathbb{N}$. Furthermore,

$$
x^{2}+2 y^{2}-2 x y-x=(x-y)^{2}+y^{2}-x \geq(x-y)^{2}+(y-x) \geq 0
$$

for all $(x, y) \in \mathbb{N}^{2}$ because $a^{2}-a \geq 0$ for all $a \in \mathbb{N}$. Thus $f_{1}(x, y, \sigma)$ is non-decreasing and it suffices to prove the statement for $\sigma^{*}=\frac{1}{2}$.

We need to prove

$$
\begin{aligned}
& \frac{1}{2} y(y-1)+\frac{1}{2}\left[x^{2}+2 y^{2}-2 x y-x\right]+\left[x^{2}-x y+2(y-x)\right] \geq 0 \\
& \Leftrightarrow \quad y(y-1)+x^{2}+2 y^{2}-2 x y-x+2 x^{2}-2 x y+4(y-x) \geq 0 .
\end{aligned}
$$

By simplifying we obtain

$$
3 x^{2}+3 y^{2}-4 x y+3 y-5 x \geq 0 \Leftrightarrow 2(x-y)^{2}+x(x-5)+y(y+3) \geq 0 .
$$

The last inequality clearly holds for all pairs $(x, y)$ with $x \geq 5$. For $x=4$, we find $2(4-y)^{2}-4+y(y+3) \geq 0$ which is true for $y \geq 1$, and for $y=0$ it can be verified by inspection. For $x=3$, we find $2(3-y)^{2}-6+y(y+3) \geq 0$ which is true for $y \geq 2$, and for $y \in\{0,1\}$ it can be verified by inspection. For $x=2$, we find $2(2-y)^{2}-6+y(y+3) \geq 0$ which again holds for $y \geq 2$, and for $y \in\{0,1\}$ it can be verified by inspection. For $x=1$, we find $2(1-y)^{2}-4+y(y+3) \geq 0$ which holds for $y \geq 1$. For $y=0$ the inequality does not hold, but this is the case $(x, y)=(1,0)$ which we explicitly excluded in the claim. For $x=0$, the inequality holds.

We now give the formal proof of Theorem 2.

Proof of Theorem 2. We first show that inequality (5) of Lemma 1 holds for the functions $\alpha(\rho, \sigma)=(2 \rho(1+\sigma)+1) /(1+$ $2 \sigma)$ and $\beta(\rho, \sigma)=(1+\rho) /(1+2 \sigma)$. That is,

$$
(1+\rho x) y-\rho(x-1) x-x \leq-\frac{1+\rho}{1+2 \sigma}(1+\sigma(x-1)) x+\frac{2 \rho(1+\sigma)+1}{1+2 \sigma}(1+\sigma(y-1)) y .
$$

Multiplying both sides by $(1+2 \sigma)$, we obtain

$$
(1+2 \sigma)[(1+\rho x) y-\rho(x-1) x-x] \leq-(1+\rho)(1+\sigma(x-1)) x+(2 \rho(1+\sigma)+1)(1+\sigma(y-1)) y,
$$

which we rewrite as $f_{1}(x, y, \sigma) \rho+f_{2}(x, y, \sigma) \geq 0$, where

$$
\begin{aligned}
f_{1}(x, y, \sigma) & =-(1+\sigma(x-1)) x+2(1+\sigma)(1+\sigma(y-1)) y+(1+2 \sigma)((x-1) x-x y) \\
& =2 y(y-1) \sigma^{2}+(-(x-1) x+2(y-1) y+2 y+2 x(x-1)-2 x y) \sigma+(-x+2 y+(x-1) x-x y) \\
& =2 y(y-1) \sigma^{2}+\left[x^{2}+2 y^{2}-2 x y-x\right] \sigma+\left[x^{2}-x y+2(y-x)\right]
\end{aligned}
$$

and

$$
\begin{aligned}
f_{2}(x, y, \sigma) & =-(1+\sigma(x-1)) x+(1+\sigma(y-1)) y+(1+2 \sigma)(x-y) \\
& =\sigma y(y-1)-\sigma x(x-1)+2 \sigma(x-y) \\
& =\left(y^{2}-x^{2}+3(x-y)\right) \sigma .
\end{aligned}
$$

We first consider the case $(x, y)=(1,0)$. In this case, we do no have $f_{1}(x, y, \sigma) \geq 0$. Substituting the values for $x$ and $y$, we obtain $-\rho+2 \sigma \geq 0$, which is true if and only if $\rho \leq 2 \sigma$.

Case i). Suppose $\frac{1}{2} \leq \sigma \leq \rho \leq 2 \sigma$. For the pair $(x, y)=(1,0)$, the inequality is true if and only if $\rho \leq 2 \sigma$. For all other pairs, we have $f_{1}(x, y, \sigma) \geq 0$, and hence

$$
f_{1}(x, y, \sigma) \rho+f_{2}(x, y, \sigma) \geq f_{1}(x, y, \sigma) \sigma+f_{2}(x, y, \sigma)
$$

meaning that is suffices to show that $f_{1}(x, y, \sigma) \sigma+f_{2}(x, y, \sigma) \geq 0$. Dividing by $\sigma$, this is equivalent to

$$
\begin{aligned}
& 2 y(y-1) \sigma^{2}+\left[x^{2}+2 y^{2}-2 x y-x\right] \sigma+\left[x^{2}-x y+2(y-x)\right]+\left(y^{2}-x^{2}+3(x-y)\right) \geq 0 \\
& \Leftrightarrow 2 y(y-1) \sigma^{2}+\left[x^{2}+2 y^{2}-2 x y-x\right] \sigma+\left[y^{2}-x y+(x-y)\right] \geq 0 .
\end{aligned}
$$

Again, we see that the terms before $\sigma^{2}$ and $\sigma$ are non-negative for all $x, y \in \mathbb{N}$ (see proof of Lemma 2). Thus, if the inequality holds for some $\sigma^{*}$, then it holds for all $\sigma \geq \sigma^{*}$. We take $\sigma^{*}=\frac{1}{2}$. Multiplying the resulting inequality by 2 , we obtain 


$$
\begin{aligned}
& y(y-1)+\left[x^{2}+2 y^{2}-2 x y-x\right]+2\left[y^{2}-x y+(x-y)\right] \geq 0 \\
& \Leftrightarrow \quad x^{2}+5 y^{2}-4 x y-3 y+x \geq 0 \\
& \Leftrightarrow \quad(x-2 y)^{2}+y(y-3)+x \geq 0 .
\end{aligned}
$$

The latter inequality holds for all $y \notin\{1,2\}$. For $y=1$, we find $(x-2)^{2}-2+x \geq 0$. The inequality clearly holds for all $x \geq 2$, and for $x \in\{0,1\}$ it can be verified by inspection. For $y=2$, we find $(x-4)^{2}-2+x \geq 0$. This is again clearly true for $x \geq 2$, and can be checked by inspection for $x \in\{0,1\}$.

Case ii). We now prove the second claim of the theorem. If $(x, y) \in \mathbb{N}^{2} \backslash\{(1,0)\}$, then $f_{1}(x, y, \sigma) \geq 0$ by Lemma 2 , meaning that $f_{1}(x, y, \sigma) \rho+f_{2}(x, y, \sigma)$ is non-decreasing in $\rho$. From the proof of Lemma 2, it follows that $f_{1}(x, y, \sigma)=0$ if and only if $(x, y) \in\{(1,1),(2,1)\}$ (which can be seen by checking all the cases). Note that this observation is independent of $\sigma$. For $(x, y) \in\{(1,1),(2,1)\}$ it also holds that $f_{2}(x, y, \sigma)=0$, which implies that $f_{1}(x, y, \sigma) \rho+f_{2}(x, y, \sigma)=0$ for every $\rho$. Therefore, we can focus on pairs $(x, y)$ for which $f_{1}(x, y, \sigma)>0$. It follows that any $\rho^{*}$ for which

$$
\rho^{*} \geq \sup _{x, y \in \mathbb{N}: f_{1}(x, y, \sigma)>0}-\frac{f_{2}(x, y, \sigma)}{f_{1}(x, y, \sigma)}
$$

yields the inequality for all $\rho \geq \rho^{*}$. It is not hard to see that this supremum is indeed finite, for every fixed $\sigma$. It can be proved that $f_{1}(x, y, \sigma) \rho^{\prime}+f_{2}(x, y, \sigma) \geq 0$ holds for some large constant $\rho^{\prime}$, which then serves as an upper bound on the supremum. For the pair $(x, y)=(0,1)$, we find $-f_{2} / f_{1}=\sigma /(1+\sigma)$, but we will see later that the supremum on the other pairs obtained is larger than $\sigma /(1+\sigma)$.

Note that by now, we can focus on pairs in $\{(x, y): x \geq 1, y \geq 2\}$, since for all other pairs we have either proven the inequality or given $-f_{2} / f_{1}$. That is, we are interested in

$$
\sup _{\{(x, y): x \geq 1, y \geq 2\}}-\frac{f_{2}(x, y, \sigma)}{f_{1}(x, y, \sigma)} .
$$

Note that $f_{2}(x, y, \sigma)=\left(y^{2}-x^{2}+3(x-y)\right) \sigma=(x+y-3)(y-x) \geq 0$ if $y \geq x$ (using that $x+y \geq 3$ for $(x, y) \in\{(x, y)$ : $x \geq 1, y \geq 2\}$ ). Hence, if $y \geq x$ we have $-f_{2} / f_{1} \leq 0$, so these pairs are not relevant for the supremum (if it follows that the upper bound on the supremum for all other pairs is positive, which we will indeed see later). Therefore, we can focus on pairs with $y<x$.

We substitute $x=a y$ for some (rational) $a>1$. Note that

$$
\sup _{a \in \mathbb{R}_{>1}} \sup _{y \geq 2}-\frac{f_{2}(a y, y, \sigma)}{f_{1}(a y, y, \sigma)}
$$

provides an upper bound on (10). Using the identities above, we have

$$
\begin{aligned}
f_{1}(a y, y, \sigma) & =\left[(1+\sigma) a^{2}-(2 \sigma+1) a+2 \sigma(\sigma+1)\right] y^{2}-\left[(2+\sigma) a+2 \sigma^{2}-2\right] y \\
-f_{2}(a y, y, \sigma) & =\left[\left(a^{2}-1\right) \sigma\right] y^{2}+[3(1-a) \sigma] y
\end{aligned}
$$

We determine an upper bound on the expression

$$
\begin{aligned}
-\frac{f_{2}(a y, y, \sigma)}{f_{1}(a y, y, \sigma)} & =\frac{\left[\left(a^{2}-1\right) \sigma\right] y^{2}+[3(1-a) \sigma] y}{\left[(1+\sigma) a^{2}-(2 \sigma+1) a+2 \sigma(\sigma+1)\right] y^{2}-\left[(2+\sigma) a+2 \sigma^{2}-2\right] y} \\
& =\frac{\left[\left(a^{2}-1\right) \sigma\right] y+[3(1-a) \sigma]}{\left[(1+\sigma) a^{2}-(2 \sigma+1) a+2 \sigma(\sigma+1)\right] y-\left[(2+\sigma) a+2 \sigma^{2}-2\right]} \\
& =\frac{\alpha y+\beta}{\gamma y-\delta}
\end{aligned}
$$

for $y \geq 2$. Elementary calculus shows that the derivative with respect to $y$ of $(12)$ is given by $-(\alpha \delta+\gamma \beta) /(\gamma y-\delta)^{2}$, which means the expression in (12) is non-decreasing or non-increasing in $y$. We have

$$
\begin{aligned}
\alpha \delta+\gamma \beta & =\left(a^{2}-1\right) \sigma\left[(2+\sigma) a+2 \sigma^{2}-2\right]+3(1-a)\left[(1+\sigma) a^{2}-(2 \sigma+1) a+2 \sigma(1+\sigma)\right] \\
& =(1-a) \sigma\left[-(1+a)\left((2+\sigma) a+2 \sigma^{2}-2\right)+3\left((1+\sigma) a^{2}-(2 \sigma+1) a+2 \sigma(1+\sigma)\right)\right] \\
& =(1-a) \sigma\left[(3(1+\sigma)-(2+\sigma)) a^{2}+\left(2-(2+\sigma)-2 \sigma^{2}-3(2 \sigma+1)\right) a+2-2 \sigma^{2}+6 \sigma(1+\sigma)\right] \\
& =(1-a) \sigma\left[(2 \sigma+1) a^{2}-\left(2 \sigma^{2}+7 \sigma+3\right) a+\left(4 \sigma^{2}+6 \sigma+2\right)\right] \\
& =(1-a) \sigma\left[(2 \sigma+1) a^{2}-(2 \sigma+1)(\sigma+3) a+(2 \sigma+1)(2 \sigma+2)\right]
\end{aligned}
$$




$$
\begin{aligned}
& =(1-a) \sigma(1+2 \sigma)\left[a^{2}-(\sigma+3) a+(2 \sigma+2)\right] \\
& =(1-a) \sigma(1+2 \sigma)\left[\left(a-\frac{\sigma+3}{2}\right)^{2}-\frac{1}{4}(1-\sigma)^{2}\right] .
\end{aligned}
$$

Before we can proceed, we need to prove the following claim.

Claim 1. The function $x_{2}=\left(\alpha x_{1}+\beta\right) /\left(\gamma x_{1}-\delta\right)$ has a vertical asymptote at $x_{1}^{*}=\delta / \gamma<2$.

Proof. It is not hard to verify that $x_{2}$ has a vertical asymptote at $x_{1}^{*}=\delta / \gamma$. Note that since $a>1$ we have $\delta>0$ for all $\sigma \geq 0$. If $\gamma<0$ then $x_{1}^{*}<0$. If $\gamma>0$, we claim that $x_{1}^{*}<2$. This is equivalent to showing that

$$
(2+\sigma) a+2 \sigma^{2}-2<2(1+\sigma) a^{2}-2(2 \sigma+1) a+4 \sigma(\sigma+1),
$$

which holds if and only if

$$
2(1+\sigma) a^{2}-(5 \sigma+4) a+2(1+\sigma)^{2}=2(1+\sigma)\left(\left[a-\frac{5 \sigma+4}{4(1+\sigma)}\right]^{2}-\frac{1}{4}\left[\frac{5 \sigma+4}{2(1+\sigma)}\right]^{2}+(1+\sigma)\right)>0 .
$$

If now suffices to show that

$$
-\frac{1}{4}\left[\frac{5 \sigma+4}{2(1+\sigma)}\right]^{2}+(1+\sigma)>0 .
$$

But this is true for all $\sigma>0$ and thus the claim follows.

We can now conclude the proof of Theorem 2 by distinguishing three cases:

Case $\sigma=1$. It follows that the expression in (13) is non-positive for all $a>1$, which implies that $-(\alpha \delta+\gamma \beta) /(\gamma y-\delta)^{2} \geq$ 0 and hence $-f_{2} / f_{1}$ is non-decreasing in $y \geq 2$ for every $a>1$ (using Claim 1). We obtain

$$
\lim _{y \rightarrow \infty}-\frac{f_{2}(a y, y, \sigma)}{f_{1}(a y, y, \sigma)}=\frac{\sigma\left(a^{2}-1\right)}{(1+\sigma) a^{2}-(2 \sigma+1) a+2 \sigma(\sigma+1)}=: h_{1}(a, \sigma)
$$

and maximizing this function over $a \in \mathbb{R}_{>1}$, we find the optimum

$$
a^{*}(\sigma)=1+\sigma+\sqrt{\sigma(\sigma+2)} \text {. }
$$

Case $\frac{1}{2} \leq \sigma<1$. More generally, for any $\sigma<1$ it holds that $\alpha \delta+\gamma \beta \leq 0$ if and only if $a \notin(1+\sigma, 2)$. In particular for every $a \notin(1+\sigma, 2)$, we can then show that

$$
\sup _{y \geq 2}-\frac{f_{2}(a y, y, \sigma)}{f_{1}(a y, y, \sigma)} \leq \lim _{y \rightarrow \infty}-\frac{f_{2}\left(a^{*} y, y, \sigma\right)}{f_{1}\left(a^{*} y, y, \sigma\right)}
$$

with $a^{*}$ as in (14) using the same argument as in the case $\sigma=1$. Claim 1 implies that if the expression (12) is nonincreasing in $y$, which is the case when $a \in(1+\sigma, 2)$, then the maximum value is attained in $y=2$. That is, we are interested in the expression $-f_{2}(2 a, 2, \sigma) / f_{1}(2 a, 2, \sigma)$, and in particular, we want to show that the supremum over $a \in(1+\sigma, 2)$ does not exceed the right hand side of (15), i.e., the supremum over all $a \notin(1+\sigma, 2)$.

Given the discussion above, it suffices to study

$$
\begin{aligned}
-\frac{f_{2}(2 a, 2, \sigma)}{f_{1}(2 a, 2, \sigma)} & =\frac{\left[\left(a^{2}-1\right) \sigma\right] 2+[3(1-a) \sigma]}{\left[(1+\sigma) a^{2}-(2 \sigma+1) a+2 \sigma(\sigma+1)\right] 2-\left[(2+\sigma) a+2 \sigma^{2}-2\right]} \\
& =\frac{\sigma\left(2 a^{2}-3 a+1\right)}{2(1+\sigma) a^{2}-(5 \sigma+4) a+2(1+\sigma)^{2}}=: h_{2}(a, \sigma)
\end{aligned}
$$

for $a \in(1+\sigma, 2)$. For $a>1$, this expression is maximized for

$$
b^{*}(\sigma)=1+\sigma+\sqrt{\sigma\left(\sigma+\frac{1}{2}\right)}
$$

which in particular gives an upper bound for $a \in(1+\sigma, 2)$.

It now suffices to show that

$$
\Delta(\sigma):=h_{1}\left(a^{*}(\sigma), \sigma\right)-h_{2}\left(b^{*}(\sigma), \sigma\right) \geq 0,
$$


since this implies that the supremum over $a>1$ in (11) is attained at some $a \notin(1+\sigma, 2)$. While unfortunately we lack an analytical proof of this inequality, it can be verified numerically (see also Fig. 4 in Appendix A).

Case $\sigma>1$. Here we can use a similar reasoning as in the previous case. The only difference is that now the expression in (12) is non-increasing for $a \in(2,1+\sigma)$, but this does not affect the above arguments because we maximize over all $a>1$ when obtaining $b^{*}(\sigma)$.

Numerical experiments suggest that $\Delta(\sigma)$ is non-negative for all $\sigma \geq \frac{1}{2}$. In Appendix A, we describe a procedure to verify this for $\sigma \in\left[\frac{1}{2}, \bar{\sigma}\right]$ for any given $\bar{\sigma} .^{7}$ We emphasize that for a fixed $\sigma$ with $\Delta(\sigma) \geq 0$, the proof that the inequality holds for all $h(\sigma) \leq \rho \leq 2 \sigma$ is exact in the parameter $\rho$. The first two cases of Theorem 2 capture all price of anarchy results from the literature.

We now show that the bound in Theorem 2 is tight for arbitrary $\rho, \sigma \geq 0$. To this aim, we generalize the lower bound construction of Christodoulou and Koutsoupias [8] for classical congestion games with $\rho=\sigma=1$. This construction is also adapted in the risk-uncertainty model by Nikolova et al. [18] and the altruism model by Chen et al. [6].

Theorem 3. For $\rho, \sigma>0$ fixed, there exists a linear congestion game such that

$$
\operatorname{PoA}(\rho, \sigma) \geq \frac{2 \rho(1+\sigma)+1}{\rho+1} .
$$

Proof. We construct a congestion game of $n \geq 3$ players and $|E|=2 n$ resources. The set $E$ is divided in the sets $E_{1}=$ $\left\{h_{1}, \ldots, h_{n}\right\}$ and $E_{2}=\left\{g_{1}, \ldots, g_{n}\right\}$. Player $i$ has two pure strategies: $\left\{h_{i}, g_{i}\right\}$ and $\left\{h_{i+1}, g_{i-1}, g_{i+1}\right\}$, where the indices appear as $i \bmod n$. The cost functions of the elements in $E_{1}$ are $c_{e}(x)=x$, whereas the cost functions of the elements in $E_{2}$ are $c_{e}(x)=\rho x$.

Regardless which strategy player $i$ plays, he always uses at least one resource from both $E_{1}$ and $E_{2}$, implying that $C_{i}^{\sigma}(s) \geq \rho+1$. This implies that

$$
C^{\sigma}(t)=\sum_{i \in N} C_{i}^{\sigma}(s) \geq(\rho+1) n
$$

for every strategy profile $t$, and in particular for a social optimum $s^{*}$.

We will now show that the strategy profile $s$ where every agent $i$ plays its second strategy $\left\{h_{i+1}, g_{i-1}, g_{i+1}\right\}$ is a Nash equilibrium. We have

$$
C_{i}^{\rho}(s)=2 \rho[1+\rho(2-1)]+1=2 \rho^{2}+2 \rho+1 .
$$

If some agent $i$ deviates to its first strategy $s_{i}^{\prime}$, we have

$$
C_{i}^{\rho}\left(s_{i}^{\prime}, s_{-i}\right)=\rho[1+\rho(3-1)]+(1+\rho(2-1))=2 \rho^{2}+2 \rho+1,
$$

since there are then three agents using $g_{i}$ and two agents using $h_{i}$. This shows that $s$ is a Nash equilibrium. The social cost of this strategy $s$ is

$$
C^{\sigma}(s)=n(1+2 \rho[1+\sigma(2-1)])=(1+2 \rho(1+\sigma)) n .
$$

Combining (19) with (18) then gives the desired result.

\subsection{PoA lower bound for symmetric network congestion games}

In this section, we show that the bound of Theorem 2 is asymptotically tight even for the special case of linear symmetric network congestion games. This improves a result for the risk-uncertainty model by Piliouras et al. [18], who prove asymptotic tightness for symmetric linear congestion games for their respective values of $\rho$ and $\sigma$ only. It also improves a result for the altruism model by Chen et al. [6], who show tightness only for general congestion games.

For the classical congestion game setting with $\rho=\sigma=1$, Christodoulou and Koutsoupias [8] showed that for symmetric affine congestion games the bound of $\frac{5}{2}$ on the price of anarchy is asymptotically tight. More recently, Correa et al. [10] proved that the bound of $\frac{5}{2}$ is tight for symmetric network affine congestion games. Our lower bound proof is a generalization of their construction.

\footnotetext{
7 We are grateful to an anonymous reviewer for suggesting this procedure.
} 


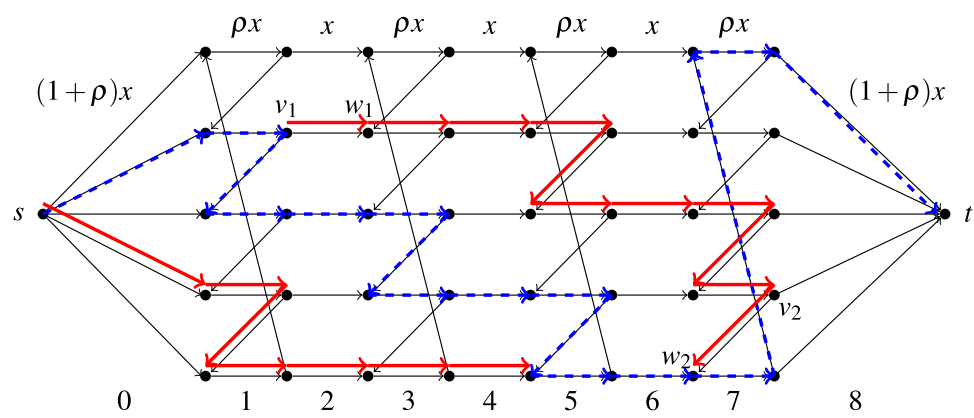

Fig. 3. Illustration of the instance for $n=5$. The dashed (blue) path indicates the strategy of player 2 in the Nash equilibrium. For every principal path, the first and last arc have cost $(1+\rho) x$, and in between the costs alternate between $\rho x$ and $x$ (starting and ending with $\rho x$ ). The diagonal connecting arcs have cost zero. The numbers at the bottom indicate the layers. The bold (red) subpaths indicate the two deviation situations that are analyzed to prove that $s$ is indeed a Nash equilibrium. (For interpretation of the colors in the figure(s), the reader is referred to the web version of this article.)

Theorem 4. For $\rho, \sigma>0$ fixed, there exists a symmetric network affine congestion game such that for every $\epsilon>0$,

$$
\operatorname{Po} A(\rho, \sigma) \geq \frac{2 \rho(1+\sigma)+1}{\rho+1}-\epsilon \text {. }
$$

Proof. We construct a symmetric network linear congestion game with $n$ players. We first describe the graph topology used in the proof of Theorem 5 in [10] (using similar notation and terminology). The graph $G$ consists of $n$ principal disjoint paths $P_{1}, \ldots, P_{n}$ from $s$ to $t$ (horizontal paths in Fig. 3 with $P_{1}$ and $P_{n}$ being the topmost and bottommost paths, respectively), each consisting of $2 n-1$ arcs (and hence $2 n$ nodes). With $e_{i, j}$ the $j$-th arc on path $i$ is denoted for $i=1, \ldots, n$ and $j=1, \ldots, 2 n-1$. Also, $v_{i, j}$ denotes the $j$-th node on path $i$ for $i=1, \ldots, n$ and $j=1, \ldots, 2 n$. There are also $n(n-1)$ connecting arcs: for every path $i$ there is an arc $\left(v_{i, 2 k+1}, v_{i+1,2 k}\right)$ for $k=1, \ldots, n$, where $i+1$ is taken modulo $n$ (the diagonal arcs in Fig. 3). For $j \geq 1$ fixed, we say that the arcs $e_{i, j}$ for $i=1, \ldots, n$ form the $(j-1)$-th layer of $G$ (see Fig. 3 ).

The cost functions are as follows. All arcs leaving $s$ (the arcs $e_{i, 1}$ for $i=1, \ldots, n$ ) and all arcs entering $t$ (the arcs $e_{i, 2 n}$ for $i=1, \ldots, n)$ have cost $c_{e}(x)=(1+\rho) x$. For all $i=1, \ldots, n$, the arcs $e_{i, 2 k-1}$ for $k=1, \ldots, n-1$ have cost function $c_{e}(x)=\rho x$, whereas the arcs $e_{i, 2 k}$ for $k=1, \ldots, n-2$ have cost function $c_{e}(x)=x$. All other arcs (the diagonal connecting arcs) have cost zero.

The feasible strategy profile $t$ in which player $i$ uses principal path $P_{i}$, for all $i=1, \ldots, n$ has social $\operatorname{cost} C^{\sigma}(t)=$ $n(2(1+\rho)+(n-1) \rho+(n-2))=n((1+\rho) n+\rho)$. A Nash equilibrium is given by the strategy profile in which every player $k$ uses the following path: she starts with arcs $e_{k, 1}$ and $e_{k, 2}$, then uses all arcs of the form $e_{k+j, 2 j}, e_{k+j, 2 j+1}, e_{k+j, 2 j+2}$ for $j=1, \ldots, n-1$, and ends with arcs $e_{k+n-1,2 n-2}, e_{k+n-1,2 n-1}$ (and uses all connecting arcs in between). ${ }^{8}$ Note that all the (principal) arcs of layer $j$ have load 1 is $j$ is even, and load 2 if $j$ is odd. The social cost of this profile is given by $C^{\sigma}(s)=n(2(1+\rho)+(n-1) \cdot 2 \cdot \rho(1+\sigma(2-1))+n-2)=n((1+2 \rho(1+\sigma)) n-2 \rho \sigma)$. It follows that $C^{\sigma}(s) / C^{\sigma}(t) \uparrow$ $(1+2 \rho(1+\sigma)) /(1+\rho)$ as $n \rightarrow \infty$. We now show that the above mentioned strategy profile $s$ is indeed a Nash equilibrium.

Fix some player, say player 2, as in Fig. 3, and suppose that this player deviates to some path $Q$. Let $j$ be the first layer in which $P_{2}$ and $Q$ overlap. Note that $j$ must be odd. The cost $C_{2}^{\rho}(s)$ of player 2 , on the subpath of $P_{2}$ leading to the first overlapping arc with $Q$, is at most

$$
(1+\rho)+\frac{j-1}{2} \cdot[2 \cdot[\rho(1+\rho(2-1))]+1]+\rho(1+\rho(2-1))=(1+\rho)^{2}+\frac{j-1}{2}(1+2 \rho(1+\rho))
$$

The subpath of $Q$ leading to the first overlapping arc with $P_{2}$ has $C_{i}^{\rho}\left(Q, s_{-i}\right)$ as follows. She uses at least one arc in every odd layer (before the overlapping layer) with a load of 3 and one arc of every even layer (before the overlapping arc) with load 2, meaning that the cost of player $i$ on the subpath of $Q$ is at least

$$
(1+\rho)(1+\rho(2-1))+\frac{j-1}{2} \cdot[(\rho(1+\rho(3-1)))+(1+\rho(2-1))]=(1+\rho)^{2}+\frac{j-1}{2} \cdot(2 \rho+1)(1+\rho)
$$

Since $1+2 \rho(1+\rho)<(2 \rho+1)(1+\rho)$ for all $\rho \geq 0$, it follows that the cost of player $i$ on the subpath of $P_{2}$ is no worse than that of the subpath of $Q$, when player 2 deviates from $P_{2}$ to $Q$. It follows that it suffices to show that $P_{2}$ is an equilibrium strategy in $s$ with respect to deviations $Q$ that overlap on the first arc $e_{2,1}$ with $P_{2}$. A similar argument shows that it also suffices to look at deviations $Q$ for which $Q$ and $P_{2}$ overlap on the last arc $e_{2,2 n-1}$ of $P_{2}$.

Now suppose that $P_{2}$ and $Q$ do not overlap on some internal part of $P_{2}$. Note that the first arc of $Q$ that is not contained in $P_{2}$, say $\left(v_{1}, w_{1}\right)$ must be in an even layer, and also that the last arc, say $\left(v_{2}, w_{2}\right)$ (which is a connecting arc) is in an

\footnotetext{
8 This is similar to the construction in [10, Theorem 5].
} 
odd layer (note that $v_{1} \neq s$ and $w_{2} \neq t$ w.l.o.g. by what is said in the previous paragraph). It is not hard to see that the subpath of $Q$ from $v_{1}$ to $w_{2}$ contains the same number of even-layered arcs as the subpath of $P_{2}$, and the same number of odd-layered arcs as the subpath of $P_{2}$. However, the load on all the odd-layered arcs on the subpath of deviation $Q$ is 3 , whereas the load on odd-layered arcs in the subpath of $P_{2}$ between $v_{1}$ and $w_{2}$ (in strategy $s$ ) is 2 . Similarly, the load on every even-layered arc on the subpath of deviation $Q$ is 2 , whereas the load on ever even-layered arc in the subpath of $P_{2}$ is 1 . Hence the subpath of deviation $Q$ between $v_{1}$ and $w_{2}$ can never be profitable.

\section{Price of stability}

In this section, we present our bounds on the price of stability for pure Nash equilibria in affine congestion games. We first establish our upper bound and show that it is tight for general congestion games (Section 4.1). We then turn to symmetric network congestion games and derive improved bounds (Section 4.2).

\subsection{PoS for general affine congestion games}

Theorem 5. We have

$$
\operatorname{PoS}(\rho, \sigma) \leq \frac{\sqrt{\sigma(\sigma+2)}+\sigma}{\sqrt{\sigma(\sigma+2)}+\rho-\sigma} \quad \text { for } \sigma>0 \text { and } \frac{2 \sigma}{1+\sigma+\sqrt{\sigma(\sigma+2)}} \leq \rho \leq 2 \sigma
$$

and this bound is asymptotically tight.

We need the following technical lemma.

Lemma 3. Let $\sigma \geq 0$ be fixed. For all non-negative integers $x$ and $y$ we have

$$
\left(x-y+\frac{1}{2}\right)^{2}-\frac{1}{4}+2 \sigma x(x-1)+(\sqrt{\sigma(\sigma+2)}+\sigma)[y(y-1)-x(x-1)] \geq 0 .
$$

Proof. The inequality is clearly true for all $y \geq x$ so we focus on the case $y<x$. By rewriting the inequality, we obtain

$$
\begin{aligned}
(1+\sigma & +\sqrt{\sigma(\sigma+2)}) y^{2}-2 x y+(1+\sigma-\sqrt{\sigma(\sigma+2)}) x^{2} \\
& -(1+\sigma+\sqrt{\sigma(\sigma+2)}) y+(1-\sigma+\sqrt{\sigma(\sigma+2)}) x \geq 0 .
\end{aligned}
$$

By multiplying both sides with $1+\sigma-\sqrt{\sigma(\sigma+2)}$ (which is non-negative for all $\sigma \geq 0$ ) and exploiting that (1+ $\sigma+$ $\sqrt{\sigma(\sigma+2)})(1+\sigma-\sqrt{\sigma(\sigma+2)})=1$, we obtain

$$
\begin{gathered}
y^{2}-2(1+\sigma-\sqrt{\sigma(\sigma+2)}) x y+(1+\sigma-\sqrt{\sigma(\sigma+2)})^{2} x^{2} \\
-y+(1+\sigma-\sqrt{\sigma(\sigma+2)})(1-\sigma+\sqrt{\sigma(\sigma+2)}) x \geq 0 .
\end{gathered}
$$

This is equivalent to

$$
\left((1+\sigma-\sqrt{\sigma(\sigma+2)}) x-y+\frac{1}{2}\right)^{2}+(1+\sigma-\sqrt{\sigma(\sigma+2)})([1+\sigma-\sqrt{\sigma(\sigma+2)}]-1) x-\frac{1}{4} \geq 0 .
$$

Define $c:=c(\sigma)=1+\sigma-\sqrt{\sigma(\sigma+2)}$. Note that $c(\sigma)$ is a bijective function from $\mathbb{R}$ to $[0,1)$. Substituting $c$ in the above inequality, we obtain for $0 \leq c<1$ the equivalent formulation

$$
\left(c x-y+\frac{1}{2}\right)^{2}+c(1-c) x-\frac{1}{4} \geq 0 .
$$

For $x=0$, the inequality reduces to $\left(\frac{1}{2}-y\right)^{2}-\frac{1}{4} \geq 0$ which is true for all $y \in \mathbb{N}$. For $x=1$, we get the equivalent formulation $(y-1)(y-2 c) \geq 0$, which is clearly true for $y=1$. For $y=0$, it follows from the fact that $c \geq 0$. For $y \geq 2$ it follows from the fact that $y-2 c \geq 0$ for all $y \geq 2$, since $0 \leq c<1$. This completes the case $x=1$.

For $x \geq 2$, we rewrite the expression (20) to

$$
x(x-1) c^{2}+2 x(1-y) c+y(y-1) \geq 0 .
$$

If $y=0$, the expression in (21) is clearly non-negative for all $x \geq 2$ and $0 \leq c<1$. For $y \geq 1$, note that $g(c)=x(x-1) c^{2}+$ $2 x(1-y) c+y(y-1)$ is a quadratic and convex function for all fixed $x$ and $y$. Therefore, in particular, for any $x$ and $y$ fixed, it suffices to show that the inequality holds for the minimizer of $g$, which is $c^{*}=(y-1) /(x-1)$ (which can be found by 
differentiating with respect to $c$ ). Note that $0 \leq c^{*}<1$ by our assumption that $y \geq 1$ and $y<x$ (made at the beginning of the proof). Substituting implies that it suffices to show that

$$
\frac{x(x-1)(y-1)^{2}}{(x-1)^{2}}+\frac{2 x(1-y)(y-1)}{x-1}+y(y-1) \geq 0 .
$$

Multiplying the expression with $(x-1)$ implies that it now suffices to show that

$$
x(y-1)^{2}-2 x(y-1)^{2}+y(y-1)(x-1) \geq 0
$$

for all $1 \leq y<x$. This is always true since

$$
\begin{aligned}
x(y-1)^{2}-2 x(y-1)^{2}+y(y-1)(x-1) & =-x(y-1)^{2}+y(y-1)(x-1) \\
& =(y-1)[-x(y-1)+y(x-1)] \\
& =(y-1)(x-y) \\
& \geq 0
\end{aligned}
$$

whenever $1 \leq y<x$. This completes the proof.

Our proof is similar to the approach used by Christodoulou, Koutsoupias and Spirakis [9] to upper bound the price of stability of $\rho$-approximate equilibria. However, for general $\sigma$ the analysis is more involved. The main technical contribution is to establish the inequality in Lemma 3. The proof of the asymptotic tightness is also based on a construction given in [9] to obtain a (non-tight) lower bound on the price of stability of approximate equilibria.

Proof of Theorem 5. Without loss of generality, we may assume that $a_{e}=1$ and $b_{e}=0$ for all resources $e \in E$. Using this, we obtain that the cost of player $i$ with respect to strategy profile $s$ is

$$
C_{i}^{\rho}(s)=\sum_{e \in s_{i}}\left(1+\rho\left(x_{e}-1\right)\right)=\sum_{e \in s_{i}}\left(x_{e}+(\rho-1)\left(x_{e}-1\right)\right) .
$$

By adapting Rosenthal's potential function (4), we obtain that

$$
\Phi^{\rho}(s):=\sum_{e \in E} \frac{x_{e}\left(x_{e}+1\right)}{2}+(\rho-1) \sum_{e \in E} \frac{\left(x_{e}-1\right) x_{e}}{2}
$$

is an exact potential for $C_{i}^{\rho}(s)$. The idea of the proof is to combine the Nash inequalities and the fact that the global minimum of $\Phi^{\rho}(\cdot)$ is a Nash equilibrium.

Let $s$ denote the global minimum of $\Phi^{\rho}$ and $s^{*}$ a socially optimal solution. Further, let $x$ and $x^{*}$ be the load profiles for $s$ and $s^{*}$, respectively. Similar to the proof of Lemma 1, by exploiting that $s$ is a Nash equilibrium we obtain

$$
\sum_{e \in E} x_{e}\left(1+\rho\left(x_{e}-1\right)\right)=\sum_{i \in N} C_{i}^{\rho}(s) \leq \sum_{i \in N} C_{i}^{\rho}\left(s_{i}^{*}, s_{-i}\right) \leq \sum_{e \in E}\left(1+\rho x_{e}\right) x_{e}^{*} .
$$

The fact that $s$ is a global optimum of $\Phi^{\rho}(\cdot)$ yields $\Phi^{\rho}(s) \leq \Phi^{\rho}\left(s^{*}\right)$, which reduces to

$$
\sum_{e \in E} \rho x_{e}^{2}+(2-\rho) x_{e} \leq \sum_{e \in E} \rho\left(x_{e}^{*}\right)^{2}+(2-\rho) x_{e}^{*} .
$$

If we can find $\gamma, \delta \geq 0$ and some $K \geq 1$, for which

$$
\begin{aligned}
& (0 \leq) \gamma\left[\rho\left(x_{e}^{*}\right)^{2}+(2-\rho) x_{e}^{*}-\rho x_{e}^{2}-(2-\rho) x_{e}\right]+\delta\left[\left(1+\rho x_{e}\right) x_{e}^{*}-x_{e}\left(1+\rho\left(x_{e}-1\right)\right]\right. \\
& \leq K \cdot x_{e}^{*}\left[1+\sigma\left(x_{e}^{*}-1\right)\right]-x_{e}\left[1+\sigma\left(x_{e}-1\right)\right],
\end{aligned}
$$

then this implies that $C^{\sigma}(s) / C^{\sigma}\left(s^{*}\right) \leq K$. We take $\delta=(K-1) / \rho$ and $\gamma=((\rho-1) K+1) /(2 \rho)$. It is not hard to see that $\delta \geq 0$ always holds. However, for $\gamma$ we have to be more careful. We will later verify for which combinations of $\rho$ and $\sigma$ the parameter $\gamma$ is indeed non-negative. Rewriting the expression in (22) yields that we have to find $K$ satisfying $K \geq f_{2}\left(x_{e}, x_{e}^{*}, \sigma\right) / f_{1}\left(x_{e}, x_{e}^{*}, \rho, \sigma\right)$, where

$$
\begin{aligned}
f_{2}\left(x_{e}, x_{e}^{*}, \sigma\right) & :=\left(x_{e}^{*}\right)^{2}-2 x_{e} x_{e}^{*}+(1+2 \sigma) x_{e}^{2}-x_{e}^{*}+(1-2 \sigma) x_{e} \\
f_{1}\left(x_{e}, x_{e}^{*}, \rho, \sigma\right) & :=(1-\rho+2 \sigma)\left(x_{e}^{*}\right)^{2}-2 x_{e} x_{e}^{*}+(1+\rho) x_{e}^{2}+(\rho-1-2 \sigma) x_{e}^{*}-(\rho-1) x_{e} .
\end{aligned}
$$

Note that this reasoning is correct only if $f_{1}\left(x_{e}, x_{e}^{*}, \rho, \sigma\right) \geq 0$. This is true because 


$$
f_{1}\left(x_{e}, x_{e}^{*}, \rho, \sigma\right)=\left(x_{e}-x_{e}^{*}+\frac{1}{2}\right)^{2}-\frac{1}{4}+(2 \sigma-\rho) x_{e}^{*}\left(x_{e}^{*}-1\right)+\rho x_{e}\left(x_{e}-1\right)
$$

is non-negative for all $x_{e}, x_{e}^{*} \in \mathbb{N}, \sigma \geq 0$ and $0 \leq \rho \leq 2 \sigma$. Furthermore, the expression is zero if and only if $\left(x_{e}, x_{e}^{*}\right) \in$ $\{(0,1),(1,1)\}$. But for these pairs the nominator is also zero, and hence the expression in (22) is satisfied for these pairs. We can write

$$
f_{2}\left(x_{e}, x_{e}^{*}, \sigma\right)=\left(x_{e}-x_{e}^{*}+\frac{1}{2}\right)^{2}-\frac{1}{4}+2 \sigma x_{e}\left(x_{e}-1\right)
$$

and therefore $f_{2} / f_{1}=\frac{A}{A+(2 \sigma-\rho) B}$, where

$$
A=\left(x_{e}-x_{e}^{*}+\frac{1}{2}\right)^{2}-\frac{1}{4}+2 \sigma x_{e}\left(x_{e}-1\right) \text { and } B=x_{e}^{*}\left(x_{e}^{*}-1\right)-x_{e}\left(x_{e}-1\right) .
$$

Note that if $\rho=2 \sigma$, we have $f_{2} / f_{1}=1$, and hence we can take $K=1$. Otherwise,

$$
\frac{A}{A+(2 \sigma-\rho) B} \leq \frac{\sqrt{\sigma(\sigma+2)}+\sigma}{\sqrt{\sigma(\sigma+2)}+\rho-\sigma}=: K \quad \Leftrightarrow \quad A+(\sqrt{\sigma(\sigma+2)}+\sigma) B \geq 0 .
$$

The inequality on the right is true by Lemma 3 .

To finish the proof, we determine the pairs $(\rho, \sigma)$ for which the parameter $\gamma$ is non-negative. This holds if and only if

$$
(\rho-1) K+1=(\rho-1) \frac{\sqrt{\sigma(\sigma+2)}+\sigma}{\sqrt{\sigma(\sigma+2)}+\rho-\sigma}+1 \geq 0 .
$$

Rewriting this inequality yields the bound on $\rho$ in the statement of the theorem.

Note that Theorem 5 does not provide the bound of 2 for uniform affine congestion games stated in Table 1 . The reason is that the bound in Theorem 5 with $\rho=\sigma$ is only valid for $\sigma \geq \frac{1}{4}$ (because otherwise the lower bound on $\rho$ is not satisfied). However, for $0 \leq \sigma \leq \frac{1}{4}$ the corresponding cost functions $c_{e}(x)=\sigma x+(1-\sigma)$ have non-negative constants and thus the price of stability for classical congestion games applies (see [4]). As a consequence, we obtain

$$
\operatorname{PoS}\left(\mathcal{A}^{\prime}\right)=\max \left\{1.577, \sup _{\sigma \geq 1 / 4}\{1+\sqrt{\sigma /(\sigma+2)}\}\right\}=2 .
$$

We next provide a lower bound on the price of stability for arbitrary non-negative pairs $(\rho, \sigma)$. The proof is similar to a construction of Christodoulou et al. [9] used to give a lower bound on the price of stability for $\rho$-approximate equilibria. The key difference is to tune some parameters in the proof with respect to the Nash definition based on the cost function $C_{i}^{\rho}(\cdot)$ rather than the $\rho$-approximate Nash equilibrium definition.

Theorem 6. For $\rho, \sigma>0$ fixed with $\rho<2 \sigma$, there exists a linear congestion game such that for every $\epsilon>0$

$$
\operatorname{PoS}(\rho, \sigma) \geq \frac{\sqrt{\sigma(\sigma+2)}+\sigma}{\sqrt{\sigma(\sigma+2)}+\rho-\sigma}-\epsilon .
$$

Proof. We describe the construction of Theorem 9 [9] (using similar notation and terminology). We have a game of $n=$ $n_{1}+n_{2}$ players divided into two sets $G_{1}$ and $G_{2}$ with size resp. $n_{1}$ and $n_{2}$. Each player $i \in G_{1}$ has two strategies: $A_{i}$ and $P_{i}$. The players in $G_{2}$ have a unique strategy $D$. The strategy profile $A=\left(A_{1}, \ldots, A_{n_{1}}, D, \ldots, D\right)$ will be the unique Nash equilibrium, and the strategy profile $P=\left(P_{1}, \ldots, P_{n_{1}}, D, \ldots, D\right)$ will be the social optimum.

We have three types of resources:

- $n_{1}$ resources $\alpha_{i}, i=1, \ldots n_{1}$, with cost function $c_{\alpha_{i}}(x)=\alpha x$. The resource $\alpha_{i}$ only belongs to strategy $P_{i}$.

- $n_{1}\left(n_{1}-1\right)$ resources $^{9} \beta_{i j}, i, j=1, \ldots, n_{1}$ with $i \neq j$, with cost function $c_{\beta_{i j}}(x)=\beta x$. The resource $\beta_{i j}$ belongs only to strategies $A_{i}$ and $P_{j}$.

- One resource $\gamma$ with cost function $c_{\gamma}(x)=x$, that belongs to $A_{i}$ for $i=1, \ldots, n_{1}$ and to $D$.

The idea is to set the parameters $\alpha$ and $\beta$ in such a way that $A$ becomes the unique Nash equilibrium. For any strategy profile $s$, there are $k$ players playing strategy $A_{i}$ and $n_{1}-k$ players playing strategy $P_{i}$ in the set $G_{1}$, for some $0 \leq k \leq n_{1}$. By

9 The proof of Theorem 9 [9] contains a typo here: it says there are $n(n-1)$ resources of this type, instead of $n_{1}\left(n_{1}-1\right)$. 
symmetry, it then suffices to look at profiles $S_{k}=\left(A_{1}, \ldots, A_{k}, P_{k+1}, \ldots, P_{n_{1}}, D, \ldots, D\right)$ for $0 \leq k \leq n_{1}$. Furthermore, the first $k$ players playing $A_{i}$ all have the same cost, and also, the $n_{1}-k$ players playing $P_{i}$ have the same cost. We can therefore focus on the costs of player 1 , denoted by $C_{A}^{\rho}(k)$, and that of player $n_{1}$, denoted by $C_{P}^{\rho}(k)$. We have

$$
\begin{aligned}
C_{A}^{\rho}(k) & =\beta(k-1)+\beta(1+\rho(2-1))\left(n_{1}-k\right)+1+\rho\left(n_{2}+k-1\right) \\
& =(\beta-\beta(1+\rho)+\rho) k+\left(-\beta+\beta(1+\rho) n_{1}+1+\rho\left(n_{2}-1\right)\right) \\
& =\rho(1-\beta) \cdot k+(1-\beta-\rho)+\beta(1+\rho) n_{1}+\rho n_{2}
\end{aligned}
$$

and

$$
\begin{aligned}
C_{P}^{\rho}(k) & =\alpha+\beta\left(n_{1}-1-k\right)+\beta(1+\rho(2-1)) k \\
& =\beta \rho \cdot k+\alpha+\beta\left(n_{1}-1\right) .
\end{aligned}
$$

We can set the parameters $\alpha$ and $\beta$ such that $C_{A}^{\rho}(k)=C_{P}^{\rho}(k-1)$, meaning that $S_{k}$ is a Nash equilibrium for every $k$ (we will create a unique Nash equilibrium in a moment), that is we take

$$
\rho(1-\beta)=\beta \rho \quad \text { and } \quad(1-\beta-\rho)+\beta(1+\rho) n_{1}+\rho n_{2}=\alpha+\beta\left(n_{1}-1\right)-\beta \rho .
$$

Note that the $-\beta \rho$ term on the far right of the second equation comes from the fact that we evaluate $C_{P}^{\rho}(\cdot)$ in $k-1$ (remember that $k$ denotes the number of players playing strategy $A_{i}$, so if a player would switch to $P_{i}$ this number decreases by 1 ). Solving the left equation leads to $\beta=1 / 2$. Inserting this in the right equation, and solving for $\alpha$, gives

$$
\alpha=\rho\left(\frac{n_{1}}{2}+n_{2}-\frac{1}{2}\right)+1 .
$$

We emphasize that $\alpha, \beta>0$ for all $\rho \geq 0$. In order to make $A$ the unique Nash equilibrium, we can slightly increase $\alpha$ such that we get $C_{A}^{\rho}(k)<C_{P}^{\rho}(k-1)$ for all $k$ (which means that $A_{i}$ is a dominant strategy for player $i$ ). Note that this increase in $\alpha$ can be arbitrary small. We have

$$
\frac{C^{\sigma}(A)}{C^{\sigma}(P)}=\frac{n_{1}\left[1+\sigma\left(n_{1}+n_{2}-1\right)+\frac{1}{2}\left(n_{1}-1\right)\right]+n_{2}\left[1+\sigma\left(n_{1}+n_{2}-1\right)\right]}{n_{1}\left[\rho\left(\frac{n_{1}+1}{2}+n_{2}-1\right)+1+\frac{1}{2}\left(n_{1}-1\right)\right]+n_{2}\left[1+\sigma\left(n_{2}-1\right)\right]} .
$$

Inserting $n_{2}=a \cdot n_{1}$ for some rational $a>0$, and sending $n_{1} \rightarrow \infty$ gives a lower bound of

$$
f(a)=\frac{2 \sigma(1+a)^{2}+1}{\rho(1+2 a)+1+2 \sigma a^{2}}
$$

on the price of stability. Optimizing over $a>0$ (this only works if $\rho<2 \sigma$ ) gives

$$
a^{*}=-\frac{1}{2}+\sqrt{\frac{1}{4}+\frac{1}{2 \sigma}}
$$

and $f\left(a^{*}\right)$ then yields the bound in the statement of the theorem.

\subsection{PoS bound for symmetric network congestion games}

We derive improved bounds on the price of stability for the special case of symmetric network congestion games. Throughout this section, we focus on the case $\sigma=1$.

Theorem 7. Let $\Gamma$ be a linear symmetric network congestion game, then

$$
\operatorname{PoS}(\Gamma, \rho, 1) \leq \begin{cases}4 /(\rho(4-\rho)) & \text { if } 0 \leq \rho \leq 1 \\ 4 /(2+\rho) & \text { if } 1 \leq \rho \leq 2 \\ (2+\rho) / 4 & \text { if } 2 \leq \rho<\infty\end{cases}
$$

In particular, if $\Gamma$ is a symmetric congestion game on an extension-paralle ${ }^{10}$ graph $G$, then the upper bounds even hold for the price of anarchy. All bounds are tight.

\footnotetext{
$\overline{10}$ A graph $G$ is extension-parallel if it consists of either (i) a single edge, (ii) a single edge and an extension-parallel graph composed in series, or (iii) two extension-parallel graphs composed in parallel.
} 
For $\rho \geq 1$, the bounds were previously shown by Caragiannis et al. [5] for the price of anarchy of singleton symmetric congestion games (which can be modeled on an extension-parallel graph).

Since any Nash equilibrium under the player $\operatorname{cost} C_{i}^{\rho}(\cdot)$ is in particular a $\rho$-approximate Nash equilibrium, we also obtain the following result.

Corollary 1 . The price of stability for $\rho$-approximate equilibria, with $1 \leq \rho \leq 2$, is upper bounded by $4 /(2+\rho)$ for linear symmetric network congestion games.

The remainder of this section is dedicated to the proof of Theorem 7 . We will refer to strategy profiles as flows, since we can interpret symmetric network congestion games as a flow problem in which players each have to route one unit of unsplittable flow from a given source to a given target. To be precise, for a graph $G=(V, E)$ and given $s, t \in V$, we write $\mathcal{P}$ for the set of all simple $s, t$-paths (the common strategy set of the players). We denote $f_{P}$ as the number of players using path $P \in \mathcal{P}$. We call $f$ a feasible (unsplittable) flow if $\sum_{P \in \mathcal{P}} f_{P}=N$, and with $f_{e}$ we denote the number of players using edge $e \in E$, that is, $f_{e}=\sum_{P \in \mathcal{P}: e \in P} f_{P}$.

We use the following result due to Fotakis [11].

Lemma 4 (Fotakis [11]). Let $\Gamma$ be a congestion game with cost functions $d_{e}$, and let $\Phi$ be an exact potential for $\Gamma$. An acyclic flow $f$ minimizes the potential function $\Phi$ if and only if

$$
\sum_{e: f_{e}>g_{e}}\left(f_{e}-g_{e}\right) d_{e}\left(f_{e}\right)-\sum_{e: f_{e}<g_{e}}\left(g_{e}-f_{e}\right) d_{e}\left(f_{e}+1\right) \leq 0
$$

for every feasible flow $g$.

The following lemma gives inefficiency results for global minima of the potential function $\Phi$ (compared to any feasible flow). Since the local minima of $\Phi$ correspond to the Nash equilibria of the game $\Gamma$, it follows that the global minima of $\Phi$ are Nash equilibria. Furthermore, Fotakis [11] shows that every Nash equilibrium of a symmetric congestion game on an extension-parallel graph is a global minimum of the potential function $\Phi$. In particular, this means that the inefficiency results in Lemma 5 hold for the price of stability of symmetric network congestion games, and the price of anarchy of symmetric extension-parallel congestion games.

Lemma 5. Let $\Gamma$ be a congestion game with cost functions $d_{e}(x)=a_{e}(1+\rho(x-1))$, and let $\Phi$ be an exact potential for $\Gamma$. Let $f$ be an acyclic flow minimizing the potential function $\Phi$, then

$$
\begin{aligned}
C^{\sigma}(f) \leq & \sum_{f_{e}>g_{e}} a_{e}\left[\left(f_{e}-1\right)\left(\rho g_{e}+(\sigma-\rho) f_{e}\right)+g_{e}\right] \\
& +\sum_{f_{e} \leq g_{e}} a_{e}\left[\left(f_{e}-1\right)\left(\rho g_{e}+(\sigma-\rho) f_{e}\right)+(1+\rho) g_{e}-\rho f_{e}\right] .
\end{aligned}
$$

Furthermore, if $h=h(\rho, \sigma)<1$ and $k=k(\rho, \sigma)$ satisfy,

$$
(x-1)(\rho y+(\sigma-\rho) x)+y \leq h \cdot x[1+\sigma(x-1)]+k \cdot y[1+\sigma(y-1)]
$$

for all non-negative integers $x>y$, and

$$
(x-1)(\rho y+(\sigma-\rho) x)+(1+\rho) y-\rho x \leq h \cdot x[1+\sigma(x-1)]+k \cdot y[1+\sigma(y-1)]
$$

for all non-negative integers $x \leq y$, then $C^{\sigma}(f) / C^{\sigma}(g) \leq k(\rho, \sigma) /(1-h(\rho, \sigma))$.

Proof. We write $d_{e}(x)=a_{e}[1+\sigma(x-1)]+a_{e}[(\rho-\sigma)(x-1)]$ in the left summation, and obtain, using Lemma 4,

$$
\begin{aligned}
\sum_{f_{e}>g_{e}} f_{e} a_{e}\left[1+\sigma\left(f_{e}-1\right)\right] \leq & \sum_{f_{e}>g_{e}} a_{e}\left(g_{e}\left[1+\rho\left(f_{e}-1\right)\right]+f_{e}(\sigma-\rho)\left(f_{e}-1\right)\right) \\
& +\sum_{f_{e}<g_{e}}\left(g_{e}-f_{e}\right) a_{e}\left(1+\rho f_{e}\right)
\end{aligned}
$$

Applying the inequality, we find 


$$
\begin{aligned}
C^{\sigma}(f)= & \sum_{f_{e}>g_{e}} f_{e} a_{e}\left[1+\sigma\left(f_{e}-1\right)\right]+\sum_{f_{e}<g_{e}} f_{e} a_{e}\left[1+\sigma\left(f_{e}-1\right)\right]+\sum_{f_{e}=g_{e}} f_{e} a_{e}\left[1+\sigma\left(f_{e}-1\right)\right] \\
\leq & \sum_{f_{e}>g_{e}} a_{e}\left[\left(f_{e}-1\right)\left(\rho g_{e}+(\sigma-\rho) f_{e}\right)+g_{e}\right] \\
& +\sum_{f_{e}<g_{e}} a_{e}\left[\left(f_{e}-1\right)\left(\rho g_{e}+(\sigma-\rho) f_{e}\right)+(1+\rho) g_{e}-\rho f_{e}\right]+\sum_{f_{e}=g_{e}} f_{e} a_{e}\left[1+\sigma\left(f_{e}-1\right)\right] \\
= & \sum_{f_{e}>g_{e}} a_{e}\left[\left(f_{e}-1\right)\left(\rho g_{e}+(\sigma-\rho) f_{e}\right)+g_{e}\right] \\
& +\sum_{f_{e} \leq g_{e}} a_{e}\left[\left(f_{e}-1\right)\left(\rho g_{e}+(\sigma-\rho) f_{e}\right)+(1+\rho) g_{e}-\rho f_{e}\right]
\end{aligned}
$$

This completes the proof.

We continue the proof of the upper bounds in Theorem 7 by showing the result in the statement for $0<\rho \leq 1$. We define

$$
h(\rho, 1)=1-\rho+\frac{\rho^{2}}{4} \quad \text { and } \quad k(\rho, 1)=1
$$

and prove the correctness of the resulting inequalities in (24) and (25) (see Lemma 6). The cases $1 \leq \rho \leq 2$ and $2 \leq \rho \leq \infty$ follow (indirectly) from Caragiannis et al. [5]. ${ }^{11}$ The authors use a similar approach as here, but only show the inequality in Lemma 4 for Nash equilibria of symmetric singleton congestion games. Nevertheless, the remainder of the analysis carries over to our model.

Lemma 6. For any integers $x, y \geq 0$ and any $\rho \in(0,1]$ we have, when $x<y$,

$$
\rho \cdot x y+(y-x) \leq \frac{\rho^{2}}{4} x^{2}+y^{2},
$$

and, when $x \geq y$,

$$
\rho \cdot x y+(1-\rho)(y-x)+(1-\rho) x^{2} \leq\left(1-\rho+\frac{\rho^{2}}{4}\right) x^{2}+y^{2} .
$$

Proof. Let $y=x+z$, where $z$ is a positive integer. Then we have

$$
\begin{aligned}
v(x, y) & =\frac{\rho^{2}}{4} x^{2}+y^{2}-\rho x y-(y-x) \\
& =\frac{\rho^{2}}{4} x^{2}+\left(x^{2}+2 x z+z^{2}\right)-\rho x(x+z)-z \\
& =\left(\frac{\rho^{2}}{4}+1-\rho\right) x^{2}+(2-\rho) x z+z(z-1) \geq 0,
\end{aligned}
$$

since $\rho \in(0,1], x \geq 0$ and $z>0$.

For the second inequality, it suffices to show that

$$
w(x, y)=\frac{\rho^{2}}{4} x^{2}+y^{2}-\rho x y-(\rho-1)(x-y) \geq 0
$$

which can be seen by leaving out the term $(1-\rho) x^{2}$ on both sides of the inequality. We first treat the case $y=0$. Then

$$
w(x, 0)=\frac{\rho^{2}}{4} x^{2}+(1-\rho) x \geq 0
$$

since $x \geq 0$ and $\rho \in(0,1]$. For $y \geq 1$, we write $a=x / y$ (for sake of notation). We have

\footnotetext{
11 The model of Carigiannis et al. [5] is equivalent to our model under the transformation $\rho=1 /(1-\zeta)$, where $\zeta$ is the model parameter of [5]. That is, the range $1 \leq \rho \leq 2$ corresponds to $\zeta \in\left[0, \frac{1}{2}\right]$, and the range $2 \leq \rho \leq \infty$ to $\zeta \in\left[\frac{1}{2}, 1\right)$.
} 


$$
\begin{aligned}
w(x, y) & =\frac{\rho^{2}}{4} x^{2}+y^{2}-\rho x y-(\rho-1)(x-y) \\
& =\frac{\rho^{2}}{4} a^{2} y^{2}+y^{2}-\rho a y^{2}+(1-\rho)(a y-y) \\
& =\left[\frac{(\rho a)^{2}}{4}-\rho a+1\right] y^{2}+(1-\rho)(a-1) y \\
& =\left[\frac{\rho a}{2}-1\right]^{2} y^{2}+(1-\rho)(a-1) y \\
& =\left[\frac{\rho x}{2 y}-1\right]^{2} y^{2}+(1-\rho)\left(\frac{x}{y}-1\right) y \geq 0,
\end{aligned}
$$

since $\rho \in(0,1], y \geq 1$ and $a \geq 1$.

It remains to show tightness of the resulting bounds. For $1 \leq \rho \leq 2$, consider an instance with two players and two resources with resp. cost functions $c_{1}(x)=x$ and $c_{2}(x)=(1+\rho+\epsilon) x$ where $0<\epsilon \ll \rho$. Then the unique Nash equilibrium is given by $\left(x_{1}, x_{2}\right)=(2,0)$, and the social optimum by $\left(x_{1}^{*}, x_{2}^{*}\right)=(1,1)$. Sending $\epsilon \rightarrow 0$ gives the desired bound of $4 /(2+\rho)$.

For $2 \leq \rho \leq \infty$, we can use the same instance as the for $1 \leq \rho \leq 2$, with the only difference that $c_{2}(x)=(1+\rho-\epsilon) x$. Then the social optimum is given by $\left(x_{1}^{*}, x_{2}^{*}\right)=(2,0)$ and the unique Nash equilibrium by $\left(x_{1}, x_{2}\right)=(1,1)$.

For the case $0<\rho \leq 1$, the lower bound is technically more involved.

Lemma 7. For every fixed (rational) $0<\rho \leq 1$, and $\epsilon>0$, there exists a symmetric singleton congestion game for which the price of stability is greater than $4 /(\rho(4-\rho))-\epsilon$.

Proof. We choose values of $n$ and $i$ so that

$$
\rho=\frac{i}{n-1}
$$

where, without loss of generality, we may assume that $i$ is even. We will construct a congestion game with $n$ agents and $1+(n-i / 2)$ resources. We let $c_{0}(x)=x$ and for $e \in\{1, \ldots, n-i / 2\}$ we define

$$
c_{e}(x)=[(1-\rho)+\rho n+\epsilon] x=[1+i+\epsilon] x, \quad \text { with } 0<\epsilon \ll \rho .
$$

A socially optimal profile $s^{*}$ is given by $x_{0}^{*}=i / 2$ and $x_{e}^{*}=1$ for $e \in\{1, \ldots, n-i / 2\}$, resulting in

$$
\begin{aligned}
C^{1}\left(s^{*}\right) & =\left(\frac{i}{2}\right)^{2}+\left(n-\frac{i}{2}\right)(1+i+\epsilon) \\
& =\frac{\rho^{2}}{4}(n-1)^{2}+\left(n-\frac{1}{2} \rho(n-1)\right)[(1-\rho)+\rho n+\epsilon] \\
& =\frac{\rho^{2}}{4}\left(n^{2}-2 n+1\right)+\left(\left(1-\frac{\rho}{2}\right) n+\frac{\rho}{2}\right)[(1-\rho)+\rho n+\epsilon] \\
& =\left[\frac{\rho^{2}}{4}+\rho\left(1-\frac{\rho}{2}\right)\right] n^{2}+\left[-\frac{\rho^{2}}{2}+(1-\rho+\epsilon)\left(1-\frac{\rho}{2}\right)+\frac{\rho^{2}}{2}\right] n+\frac{\rho^{2}}{4}+\frac{\rho}{2}(1-\rho+\epsilon) \\
& =\left[\rho\left(1-\frac{\rho}{4}\right)\right] n^{2}+\left[(1-\rho+\epsilon)\left(1-\frac{\rho}{2}\right)\right] n+\frac{\rho}{2}\left(1-\frac{\rho}{2}+\epsilon\right)
\end{aligned}
$$

The unique Nash equilibrium is given by the strategy profile $s$ for which $x_{0}=n$ and $x_{e}=0$ for $e \in\{1, \ldots, n-i / 2\}$, since the perceived cost on resource $e=0$ is then precisely $(1-\rho)+\rho n$, so no player can strictly improve its (perceived) cost by deviating to one of the other resources, which have cost $(1-\rho)+\rho n+\epsilon$. The social cost of this equilibrium is $n^{2}$, which implies that

$$
\frac{C^{1}(s)}{C^{1}\left(s^{*}\right)}=\frac{n^{2}}{\left[\rho\left(1-\frac{\rho}{4}\right)\right] n^{2}+\left[(1-\rho)\left(1-\frac{\rho}{2}+\epsilon\right)\right] n+\frac{\rho}{2}\left(1-\frac{\rho}{2}+\epsilon\right)} \rightarrow \frac{1}{\rho(1-\rho / 4)}
$$

as $n \rightarrow \infty$ (note that this also means that $i \rightarrow \infty$ since $\rho$ is fixed). 


\section{Implications and additional insights}

We discuss a few implications of our bounds for the applications mentioned above (see Section 2) and provide some additional insights.

Altruism [5,6]. For $\sigma=1$, our bound $(4 \rho+1) /(\rho+1)$ reveals that the price of anarchy is increasing in $\rho$, which is counterintuitive and not desirable especially in the context of altruism or taxes. A natural question that arises is whether there exist collections $\mathcal{H}$ of congestion games for which the price of anarchy is non-increasing as a function of $\rho$.

The next theorem gives a sufficient condition for a class of instances to have the property that the price of anarchy is non-increasing in $\rho$.

Theorem 8. Let $\mathcal{H}$ be a collection of congestion games. If $\operatorname{PoA}(\mathcal{H}, 2,1)=1$, then $\operatorname{PoA}(\mathcal{H}, \rho, 1)$ is a non-increasing function for $1 \leq$ $\rho \leq 2$.

Intuitively, for $\rho=2$ it can be shown that the social optimum becomes a Nash equilibrium under the player cost function $C_{i}^{\rho}(s)$, which implies that the price of stability is 1 . Still it might happen that worse Nash equilibria arise as well. The condition $\operatorname{PoA}(\mathcal{H}, 2,1)=1$ restricts to cases where this does not happen. Even stronger, this condition ensures that all Nash equilibria become social optima; we say that the social optima are strongly enforceable. The above theorem thus states that if the social optima of the game with $\rho=2$ are strongly enforceable, then the price of anarchy $\operatorname{PoA}(\mathcal{H}, \rho, 1)$ is non-increasing in the range $1 \leq \rho \leq 2$.

In a technical report, Singh [21] shows that the social optimum is strongly enforceable for symmetric network congestion games on series-parallel graphs. We can therefore conclude that the (altruistic) price of anarchy will be a non-increasing function of $\rho$. This is a remarkable result since, to the best of our knowledge, the classical price of anarchy is unknown (the best lower bound is given by Fotakis [11]).

Proof of Theorem 8. Suppose that $\operatorname{PoA}(\mathcal{H}, \rho, 1)=: \operatorname{PoA}(\rho)$ is not non-increasing, then there exist $x<y \in[1,2]$ such that $\operatorname{PoA}(y)>\operatorname{PoA}(x)$. By using the assumption that $\operatorname{PoA}(2)=1$, we conclude that $\operatorname{PoA}(x) \geq 1=\operatorname{PoA}(2)$ because the price of anarchy is always lower bounded by 1 . Note that this also implies that $y \neq 2$. This means that

$$
\max \{\operatorname{PoA}(x), \operatorname{PoA}(2)\}<\operatorname{PoA}(y) .
$$

However, if we write $y=\gamma \cdot x+(1-\gamma) \cdot 2$ for some $\gamma \in[0,1]$, then Theorem 10.2 in [6] states that

$$
\operatorname{PoA}(y) \leq \max \{\operatorname{PoA}(x), \operatorname{PoA}(2)\}
$$

which is a contradiction.

Universal taxes [4]. Caragiannis et al. [4] show that the price of anarchy can be decreased to 2.155 through the usage of universal tax functions $\tau(\rho)=\frac{3}{2} \sqrt{3}-2$. This is a significant improvement of the price of anarchy bound of $\frac{5}{2}$ for classical congestion games. Further, from [4, Theorem 3.7] it follows that the price of anarchy can never be better than 2.155 for $0 \leq \rho \leq h(1)$. Note that the choice $\rho=h(1)$ in fact provides the best price of anarchy as indicated in Fig. 2 . On the other hand, our results show that the price of stability increases from 1.577 for classical congestion games to 2.013 for this specific set of tax functions.

Risk sensitivity under uncertainty [18]. We do not only re-obtain the price of anarchy results for risk-neutral players and players applying Wald's minimax principle (worst-case players), but our results also give a tight bound for any convex combination (in terms of player costs) of risk-neutral and worst-case risk attitudes. Furthermore, we also obtain tight price of stability results for this model.

Approximate Nash equilibria [9]. For $\sigma=1$ and $1 \leq \rho \leq 2$, we obtain a bound of $(\sqrt{3}+1) /(\sqrt{3}+\rho-1)$ on the price of stability. In particular, this also yields the same bound on the price of stability for $\rho$-approximate equilibria. This bound was previously obtained by Christodoulou et al. [9]. Conceptually our approach is different: We prove our bound by observing that every Nash equilibrium in our framework yields an approximate equilibrium. In particular, this gives rise to a potential function that can be used to carry out the technical details (namely the potential function that is exact for our congestion game). ${ }^{12}$

\footnotetext{
12 Nevertheless, the framework of Christodoulou et al. [9] is somewhat more general and might be used to obtain a tight bound for the price of stability of approximate equilibria (which is not known to the best of our knowledge).
} 


\section{Conclusion}

We introduced a new model of affine congestion games by parameterizing both the cost functions of the players and the social cost function. Our model encompasses several extensions of Rosenthal's (classical) congestion games which were previously studied in the literature. We derived bounds on the price of anarchy and the price of stability which are tight for a large range of parameters $\rho$ and $\sigma$. Our work reveals that tight bounds on the inefficiency of these extensions can be derived in a unifying manner. The study of such parameterized games seems particularly valuable if tight bounds can be derived.

A first natural extension of our model is to go beyond affine cost functions. Some of the connections between perceptionparameterized congestion games and other models revealed in this paper, continue to hold for more general cost functions (although not always as clean as for the affine case). Another natural direction for future research is to consider parameterized versions of other fundamental games such as cost sharing games, utility games, network design games or auctions.

For non-atomic network routing games [22] several extensions which were recently studied in the literature can also be unified; in particular, there are close connections between the extensions considered in $[2,7,9,12,15,16]$. Similar to the viewpoint adopted here, these extensions can be viewed as network routing games where the cost functions of the players are suitably parameterized. In fact, many of these models incorporate (implicitly or explicitly) some scaled marginal tolls into the cost functions of the players. Further, this also connects to the notion of approximate Nash equilibria (as in [9]). Several of these works find similar inefficiency bounds which can also be derived in a unifying manner by using these scaled marginal tolls.

In a recent work [13], we showed that the bound on the price of stability for symmetric network congestion games by Fotakis [11] also extends to a more general class of combinatorial congestion games, called polytopal congestion games. In particular, our results in Section 4.2 can also be applied to these games.

In this paper, we focused on the homogeneous player case because this is the setting addressed in most previous studies which we unify here. An interesting direction for future research is to consider heterogenous players. In this context, Chen et al. [6] derived a price of anarchy bound for their altruistic congestion games which depends on the extreme values of the parameter $\rho$ used by players (with $\sigma=1$ ).

\section{Acknowledgements}

We thank the anonymous reviewers for their valuable comments on the paper.

\section{Appendix A. Numerical verification of non-negativity of $\Delta(\sigma)$ as in Theorem 2}

The plot in Fig. 4 shows our numerical verification of the inequality

$$
\Delta(\sigma):=h_{1}\left(a^{*}(\sigma), \sigma\right)-h_{2}\left(b^{*}(\sigma), \sigma\right) \geq 0
$$

used in the proof of Theorem 2 for the range $\frac{1}{2} \leq \sigma \leq 2$.

We next describe a procedure that can be used to verify this inequality for $\sigma \in\left[\frac{1}{2}, \bar{\sigma}\right]$ for any fixed $\bar{\sigma} .{ }^{13}$ By solving $a^{*}(\sigma)$ in (14) for $\sigma$ we obtain

$$
\sigma=\frac{(a-1)^{2}}{2 a}
$$

and substituting this into $h_{1}\left(a^{*}(\sigma), \sigma\right)$ we obtain a simplified function which only depends on $a$ :

$$
h_{1}(a)=\frac{a(a-1)^{2}}{a^{3}-a^{2}+2 a-1} .
$$

By taking the derivative, we can verify that this function is strictly increasing in the relevant range $\left[\frac{1}{2}(3+\sqrt{5}), 1+\bar{\sigma}+\right.$ $\left.\sqrt{\bar{\sigma}^{2}+2 \bar{\sigma}}\right]$ (corresponding to the interval $\left[\frac{1}{2}, \bar{\sigma}\right]$ ). Note also that $a^{*}(\sigma)$ (as a function of $\sigma$ ) is increasing in $\sigma$. As a consequence, we conclude that $h_{1}$ (as a function of $\sigma$ and $a^{*}(\sigma)$ ) is increasing for $\sigma \in\left[\frac{1}{2}, \bar{\sigma}\right]$.

Similarly, following the same line of arguments for $h_{2}$, by solving $b^{*}(\sigma)$ in (17) for $\sigma$ we obtain

$$
\sigma=\frac{2 b^{2}-4 b+2}{4 b-3}
$$

and substituting this into $h_{2}\left(b^{*}(\sigma), \sigma\right)$ we obtain a simplified function which only depends on $b$ :

$$
h_{2}(b)=\frac{4 b^{2}-7 b+3}{4 b^{2}-b-1} \text {. }
$$

13 We are grateful to an anonymous reviewer for this suggestion. 


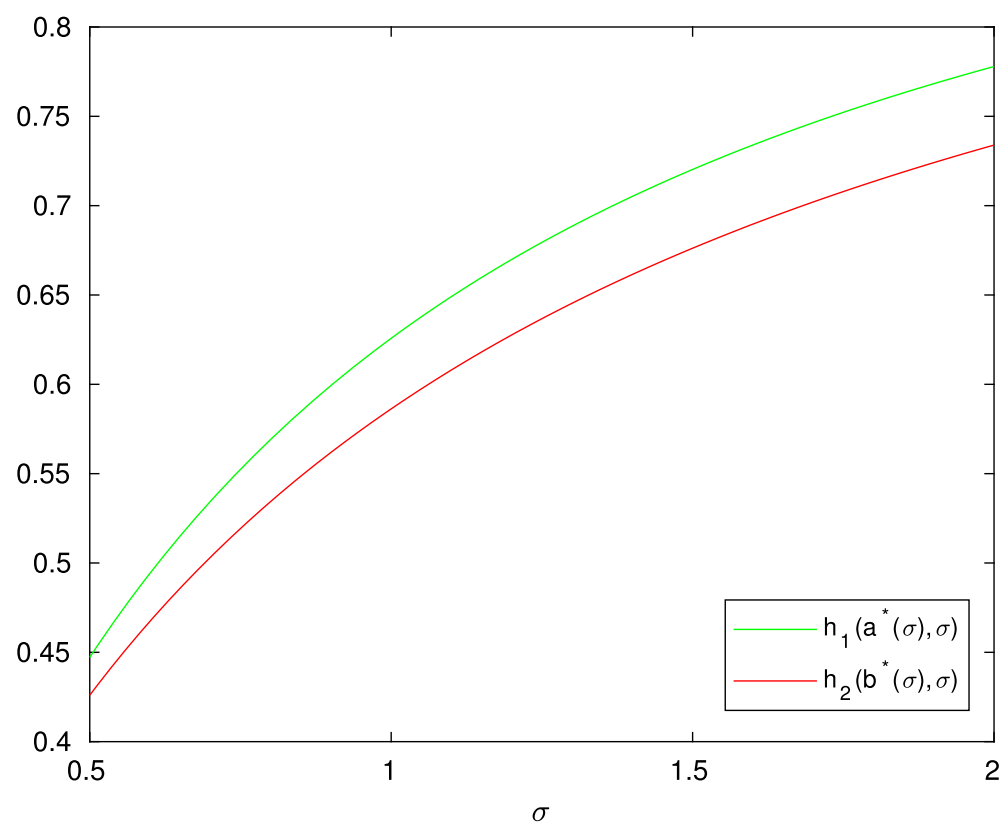

Fig. 4. Numerical verification that $h_{2}\left(b^{*}(\sigma), \sigma\right) \leq h_{1}\left(a^{*}(\sigma), \sigma\right)$ for $\frac{1}{2} \leq \sigma \leq 2$ with step size $\Delta=10^{-6}$.

By taking the derivative, we can verify that this function is strictly increasing in the relevant range

$$
\left[\frac{3}{2}+\frac{1}{\sqrt{2}}, 1+\bar{\sigma}+\sqrt{\bar{\sigma}\left(\bar{\sigma}+\frac{1}{2}\right)}\right]
$$

(corresponding to the interval $\left[\frac{1}{2}, \bar{\sigma}\right]$ ). As before, $b^{*}(\sigma)$ (as a function of $\sigma$ ) is increasing in $\sigma$ and we conclude that $h_{2}$ (as a function of $\sigma$ and $\left.b^{*}(\sigma)\right)$ is an increasing function for $\sigma \in\left[\frac{1}{2}, \bar{\sigma}\right]$.

We now argue that it is sufficient to check (26) for finitely many points in the interval $\left[\frac{1}{2}, \bar{\sigma}\right]$ in order to conclude that (26) is true for all points in this range. We use the following simple observation.

Proposition 1. Let $c \leq d$ be given. Let $p, q:[c, d] \rightarrow \mathbb{R}$ be two non-decreasing functions with the property that $p(d) \leq q(c)$. Then $p(y) \leq q(y)$ for all $y \in[c, d]$.

Let $\epsilon>0$ and define $y_{k}=\frac{1}{2}+\epsilon \cdot k$ for $k=0, \ldots,\left(\bar{\sigma}-\frac{1}{2}\right) / \epsilon$. If we can choose $\epsilon$ small enough so that $h_{2}\left(y_{k+1}\right) \leq h_{1}\left(y_{k}\right)$ holds for all $k=0, \ldots,\left(\bar{\sigma}-\frac{1}{2}\right) / \epsilon$, then we can use Proposition 1 to conclude that (26) is true for all $y \in\left[\frac{1}{2}, \bar{\sigma}\right]$ (exploiting that $h_{1}$ and $h_{2}$ are strictly increasing). For the case $\bar{\sigma}=2$ this follows from the numerical verification in Fig. 4 .

\section{References}

[1] E. Anshelevich, A. Dasgupta, J. Kleinberg, E. Tardos, T. Wexler, T. Roughgarden, The price of stability for network design with fair cost allocation, in: Proceedings of the 45th Annual IEEE Symposium on Foundations of Computer Science, FOCS '04, IEEE Computer Society, Washington, DC, USA, 2004, pp. 295-304.

[2] V. Bonifaci, M. Salek, G. Schäfer, On the efficiency of restricted tolls in network routing games, in: Lecture Notes in Computer Science, 2011.

[3] I. Caragiannis, A. Fanelli, N. Gravin, A. Skopalik, Computing approximate pure Nash equilibria in congestion games, ACM SIGecom Exch. 11 (1) (Jun 2012) 26-29, https://doi.org/10.1145/2325713.2325718.

[4] I. Caragiannis, C. Kaklamanis, P. Kanellopoulos, Taxes for linear atomic congestion games, ACM Trans. Algorithms 7 (1) (Dec 2010) 13:1-13:31, https:// doi.org/10.1145/1868237.1868251.

[5] I. Caragiannis, C. Kaklamanis, P. Kanellopoulos, M. Kyropoulou, E. Papaioannou, The Impact of Altruism on the Efficiency of Atomic Congestion Games, Springer, Berlin, Heidelberg, 2010, pp. 172-188.

[6] P.A. Chen, B. de Keijzer, D. Kempe, G. Schäfer, Altruism and its impact on the price of anarchy, ACM Trans. Econ. Comput. 2 (4) (Oct 2014) 17:1-17:45, https://doi.org/10.1145/2597893.

[7] P.A. Chen, D. Kempe, Altruism, selfishness, and spite in traffic routing, in: Proceedings of the 9th ACM Conference on Electronic Commerce, ACM, 2008, pp. $140-149$.

[8] G. Christodoulou, E. Koutsoupias, The price of anarchy of finite congestion games, in: Proceedings of the Thirty-Seventh Annual ACM Symposium on Theory of Computing, STOC '05, ACM, New York, NY, USA, 2005, pp. 67-73.

[9] G. Christodoulou, E. Koutsoupias, P.G. Spirakis, On the performance of approximate equilibria in congestion games, Algorithmica 61 (1) (Sep 2011) $116-140$.

[10] J. Correa, J. de Jong, B. de Keijzer, M. Uetz, The curse of sequentiality in routing games, in: Proceedings of the 11th International Conference on Web and Internet Economics, WINE 2015, in: Lecture Notes in Computer Science, vol. 9470, Springer-Verlag, New York, NY, USA, 2015, pp. 258-271. 
[11] D. Fotakis, Congestion games with linearly independent paths: convergence time and price of anarchy, Theory Comput. Syst. 47 (1) (Jul 2010) 113-136.

[12] D. Fotakis, D. Kalimeris, T. Lianeas, Improving selfish routing for risk-averse players, in: Web and Internet Economics - 11th International Conference. Proceedings, WINE 2015, Amsterdam, The Netherlands, December 9-12, 2015, pp. 328-342.

[13] P. Kleer, G. Schäfer, Potential function minimizers of combinatorial congestion games: Efficiency and computation, in: Proceedings of the 2017 ACM Conference on Economics and Computation, EC '17, ACM, New York, NY, USA, 2017, pp. 223-240.

[14] E. Koutsoupias, C. Papadimitriou, Worst-case equilibria, in: Proceedings of the 16th Annual Conference on Theoretical Aspects of Computer Science, STACS'99, Springer-Verlag, Berlin, Heidelberg, 1999, pp. 404-413.

[15] R. Meir, D. Parkes, Playing the wrong game: smoothness bounds for congestion games with behavioral biases, ACM SIGMETRICS Perform. Eval. Rev. 43 (3) (Nov 2015) 67-70.

[16] R. Meir, D.C. Parkes, Congestion games with distance-based strict uncertainty, arXiv:1411.4943, 2014

[17] D. Monderer, L.S. Shapley, Potential games, Games Econom. Behav. 14 (1) (1996) 124-143.

[18] G. Piliouras, E. Nikolova, J.S. Shamma, Risk sensitivity of price of anarchy under uncertainty, in: Proceedings of the Fourteenth ACM Conference on Electronic Commerce, EC '13, ACM, New York, NY, USA, 2013, pp. 715-732.

[19] R.W. Rosenthal, A class of games possessing pure-strategy Nash equilibria, Internat. J. Game Theory 2 (1973) 65-67.

[20] T. Roughgarden, Intrinsic robustness of the price of anarchy, J. ACM 62 (5) (2015) 32.

[21] C. Singh, Marginal cost pricing in atomic congestion games, Technical report.

[22] J.G. Wardrop, Some theoretical aspects of road traffic research, in: Proceedings of the Institution of Civil Engineers, vol. 1, 1952, pp. 325-378, http:// ci.nii.ac.jp/naid/10013551651/en/. 